\title{
Microflares and the Statistics of X-ray Flares
}

\author{
I. G. Hannah ${ }^{1}$, H. S. Hudson ${ }^{2}$, M. Battaglia ${ }^{1}$, \\ S. Christe ${ }^{3}$, J. Kašparová ${ }^{4}$, S. Krucker ${ }^{2}$, \\ M. R. Kundu ${ }^{5}$, and A. Veronig 6
}

\begin{abstract}
This review surveys the statistics of solar X-ray flares, emphasising the new views that RHESSI has given us of the weaker events (the microflares). The new data reveal that these microflares strongly resemble more energetic events in most respects; they occur solely within active regions and exhibit high-temperature/nonthermal emissions in approximately the same proportion as major events. We discuss the distributions of flare parameters (e.g., peak flux) and how these parameters correlate, for instance via the Neupert effect. We also highlight the systematic biases involved in intercomparing data representing many decades of event magnitude. The intermittency of the flare/microflare occurrence, both in space and in time, argues that these discrete events do not explain general coronal heating, either in active regions or in the quiet Sun.
\end{abstract}

Keywords Sun - Flares - X-rays

\section{Contents}

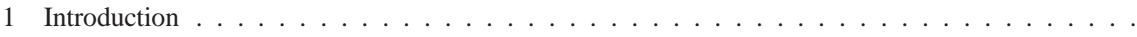

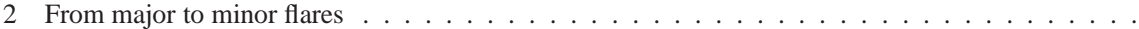

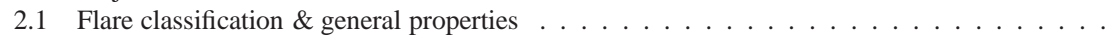

2.1.1 Flare relationship to CMEs and SEPs . . . . . . . . . . . . . . . . . .

2.2 Microflares . . . . . . . . . . . . . . . . . . . .

2.2.1 Association with active regions . . . . . . . . . . . . . . . .

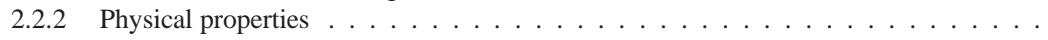

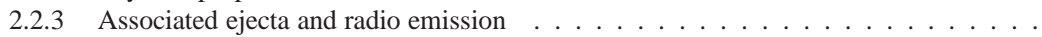

2.3 Nanoflares and non-active-region phenomena . . . . . . . . . . . . . . . .

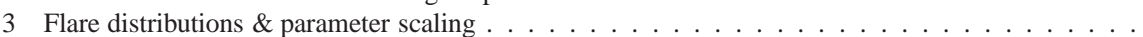

3.1 X-ray flux distributions . . . . . . . . . . . . . . . . . . .

3.2 Time distributions . . . . . . . . . . . . . . . . . . .

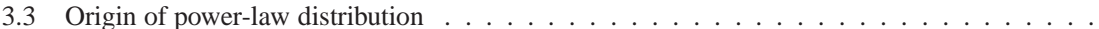

3.4 Nonthermal and thermal spectral parameters . . . . . . . . . . . . . . .

3.4 .1 Nonthermal . . . . . . . . . . . . . . . . . . . . .

${ }^{1}$ School of Physics \& Astronomy, University of Glasgow, Glasgow, G12 8QQ, UK

${ }^{2}$ Space Sciences Laboratory, University of California at Berkeley, Berkeley, CA, 94720-7450, USA

${ }^{3}$ NASA Goddard Space Flight Center, Greenbelt, MD 20771, USA

${ }^{4}$ Astronomický ústav AV ČR, v.v.i., Fričova 298, Ondřějov, 251 65, Czech Republic

${ }^{5}$ Dept. of Astronomy, Univ. of Maryland, College Park, MD 20740

${ }^{6}$ Institute of Physics/IGAM, University of Graz, Universitätsplatz 5, 8010, Graz, Austria 


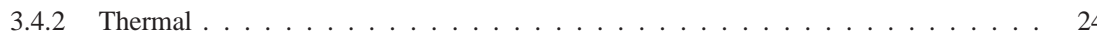

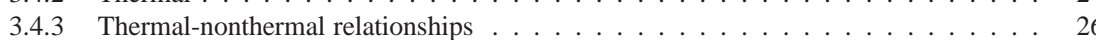

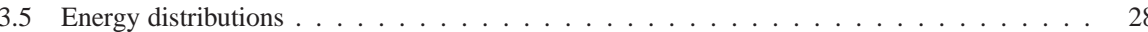

4 Conclusions \& discussion . . . . . . . . . . . . . . . . . . . . . . . . . . . . . . . 31

A Determining distribution parameters ...................... 31

\section{Introduction}

A solar flare is a rapid and transient release of energy in the solar corona, associated with electromagnetic radiation from radio waves to $\gamma$-rays, local plasma heating to tens of MK, particles accelerated to $\mathrm{GeV}$, violent mass plasma motions, and shock waves. In the largest events, a spectacular variety of phenomena can be studied in detail across many wavelength ranges to try to understand the processes involved (see examples in Fletcher et al. 2011). The X-ray emission observed is of particular interest as it shows the accelerated particles and intense heating, and therefore provides the most direct insights into the physics of the basic energy release. Hard X-rays (HXRs), from about $10 \mathrm{keV}$ to hundreds of keV, are primarily produced via thick-target bremsstrahlung (Brown 1971; Kontar et al. 2010) in which the coronal accelerated electrons are stopped instantaneously through Coulomb collisions with denser material in the lower solar atmosphere. Soft X-rays (SXRs), typically the component below tens of $\mathrm{keV}$, are thermal emission (lines and continua) by plasma of a few to tens of MK. This emission results to some extent from heating at the site of energy release, but mainly from new coronal material evaporated from the chromosphere during the impulsive phase (see Fletcher et al. 2011). Given the wealth of X-ray flare data, it is possible to study large samples of these events, allowing the statistics of the events to provide clues to the underlying processes behind the emission. This article reviews such flare studies, the results, interpretation and limitations, from a predominantly X-ray viewpoint. In particular, we discuss the advances that have been made in extending this analysis to weaker HXR events (microflares) using the Reuven Ramaty High Energy Solar Spectroscopic Imager (RHESSI) (Lin et al. 2002).

One of the most remarkable features of flare surveys is the range of magnitudes these transient phenomena span. The observed SXR flux extends over five orders of magnitude (see Figure 1.1) and over nine orders of magnitude in energy (see Figure 2.1, we return to discuss these Figures in detail in Section 3). The observations come from many spacecraft: e.g., Orbiting Solar Observatory (OSO-3) and (OSO-7), the Solar Maximum Mission (SMM), the International Cometary Explorer (ICE, a.k.a. ISEE-3), the Compton GammaRay Observatory (CGRO), Yohkoh, the Solar Heliophysical Observatory (SOHO), GRANAT/ WATCH, and the Transition Region and Coronal Dynamics Explorer (TRACE). The distributions of these and other flare parameters, such as emission at other wavelengths and for other durations, have the further striking property that they all can be well represented by a power law of the form

$$
f(x ; \alpha)=C x^{-\alpha},
$$

where $f$ is the probability density function (PDF; this is often called the flare frequency distribution) of the flare parameter $x, \alpha>0$ is the power-law index and $C$ is a scaling constant. In the case of the flare's energy $U$, the quantity $f(U) d U$ is the fraction of events per unit time releasing energy between $U$ and $U+d U$. This power-law nature of the frequency distribution has long been observed, first noticed in solar radio bursts by Akabane (1956). The fact that so many of the flare characteristics have such a distribution is thought to arise from the corona being in a self-organized critical state (Lu \& Hamilton 1991), discussed further 


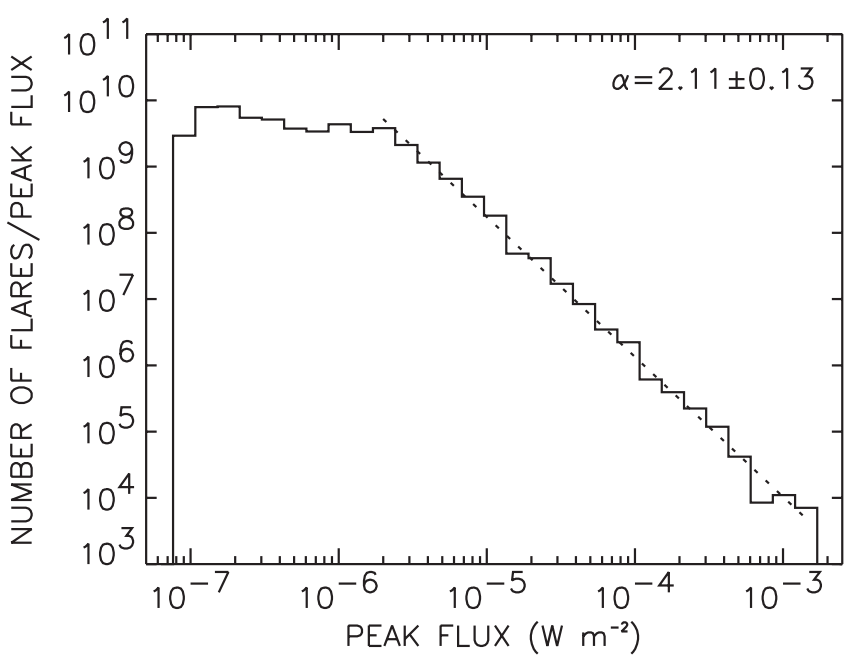

Fig. 1.1 Frequency distribution of the peak 1-8 $\mathrm{AXR}$ GOES flux of 49,409 flares between 1976 and 2000 Veronig et al. 2002a). Note that this study did not include the A-class events $\left(<10^{-7} \mathrm{~W} \mathrm{~m}^{-2}\right)$. In addition, there was no attempt at background subtraction, so the resulting value of $\alpha$ should be regarded as an upper limit (background predominantly affects smaller flares, artificially steepening the distribution).

in Section 3.3 Here, the underlying energy release from the largest to the smallest flares occurs as an "avalanche," similar to the theory for earthquake occurrence or stress relief in general (Katz 1986). These properties have brought forth interesting interpretations. The power-law distribution means that small flares are more numerous.

Could these weaker events at the currently unobservable "nanoflare" magnitude level (about $10^{-9}$ times the energy of a large flares) directly constitute the hot corona, thus explaining its heating? This is often called Parker's nanoflare mechanism (Parker 1988). The answer to this question requires knowledge of the total power that is contained in the flare distribution, i.e.

$$
P=\int_{U_{\min }}^{U_{\max }} f(U ; \alpha) U d U=\frac{C}{2-\alpha}\left[U_{\max }^{2-\alpha}-U_{\min }^{2-\alpha}\right]
$$

Since $U_{\min } \ll U_{\max }$ by definition, we can see that in the case $\alpha>2$ in Equation (1.2) the low-energy half of the distribution would contain the most energy (Hudson 1991b). In this situation the smallest flares fit the requirement for heating the corona as they have a high occurrence rate and release more net energy than the large flares. Therefore, to understand the role of flares in coronal heating, the power-law index $\alpha$ has to be accurately estimated; this is problematic as the energy is inferred from observations and thus subject to potentially large errors and biases. In addition, Equation (1.2) assumes that the distributions continue into the unobservable low-energy range. Even if the deduced $\alpha$ were large enough (i.e., larger than two), that would not automatically mean that small flares heat the corona. For further proof we would require observations showing the power-law index to maintain its value down to the distribution's physical limit, rather than its observational limit.

Between the largest flares and these theoretical nanoflares lie the microflares (nominally with energies of order $10^{-6}$ times those of large flares), and it is with these events that RHESSI's HXR observations have made major advances. RHESSI's continuous spectral coverage, down to $3 \mathrm{keV}$, uniquely allows the study of the transition between the thermal and nonthermal emissions with the same instrument. In particular, RHESSI's view of their nonthermal characteristics has allowed us to study smaller-scale active region events with simpler structures than the major events that often attract the most attention. Perhaps these 
can help us to isolate the essential physics, which could then be applied in more complex situations.

We begin this article with a general overview of flares and flare-like brightenings across the range of magnitudes (Section 2). This discloses similar properties between major and minor events, but systematic differences do occur. In Section 3 we learn what we can from these differences about the physics, taking advantage of the very large numbers of events in major surveys, including RHESSI's new HXR views. We also discuss in this section the biases that arise in the statistical surveys and possible methods for obtaining the unbiased intrinsic distributions. In Section 3.3 we briefly discuss how the power-law nature of the flare parameters arises. Conclusions and discussions are given in Section 4

\section{From major to minor flares}

\subsection{Flare classification \& general properties}

The most powerful ordinary flares have energies estimated at above $10^{33}$ ergs and present a spectacular range of phenomena, easily observed across the wavelengths. The first flare observed was a powerful event in 1859 , detectable through its small, intense white-light emission patches as described by Carrington (1859) and corroborated by Hodgson (1859). Remarkable terrestrial effects accompanied this flare and also followed it after an interval of half a day. This event anticipated much of the complexity of flares as we know them today, but it was not until the 1940s that "flare" was accepted as the term to describe these transient phenomena (Newton 1943; Richardson 1944). Events with total energy about a millionth smaller than large flares (about $10^{27} \mathrm{erg}$ ), became known as "microflares" (Schadee et al. 1983; Lin et al. 1984). Parker hypothesized that even smaller flares, "nanoflares," with energies of order one billionth of large flares or about $10^{24} \mathrm{erg}$, could be the basic unit of a localized impulsive energy release (Parker 1988).

Quantitative flare classification is based on the 1-8 $\AA$ SXR flux observed by GOES. Large flares have $\mathrm{X} n$-class, indicating a peak flux of $n \times 10^{-4} \mathrm{~W} \mathrm{~m}^{-2}$, the largest events being above X10. This classification decreases through the decades of M, C and B-class flares down to the smallest An-class events with $n \times 10^{-8} \mathrm{~W} \mathrm{~m}^{-2}$ and the sensitivity limit of the detector. The classification of flares and the associated range of GOES fluxes is shown in Table 2.1. The largest GOES flare was SOL2003-11-04T19:53 (X17.4), which saturated the detectors at $18 \times 10^{-4} \mathrm{~W} \mathrm{~m}^{-2}$ (i.e., class X18). It is estimated that this flare was an X28 event with range X25 to X31 (Kiplinger \& Garcia 2004). In terms of GOES classification, the Carrington flare appears to have been a large soft X-ray flare of magnitudfe $>$ X10 (Tsurutani et al. 2003) and like many large flares resulted in a major geomagnetic storm (Cliver \& Svalgaard 2004). This region also produced one of the largest solar energetic proton fluences at the Earth in the last 500 years (McCracken et al. 2001). These most energetic flares occur in active regions, often when new flux emerges into an already-complex magnetic structure. From the start of the GOES observations in 1975, to the start of cycle 24 in 2009, 22 flares $>$ X10 had been observed. In total, 359 GOES X-class flares, 4708 M-class, 32784 C-class and 11558 B-class flares occurred between 1976 and 2000 (Veronig et al. 2002a). Although more small flares are expected due to the inherent power-law nature of the flare distribution, they are hidden by the higher background during active times. The majority of large flares seem to occur during the peak of the solar cycle heading into the decay phase, though this behavior can vary dramatically from cycle to cycle (e.g., Hudson 2007). 


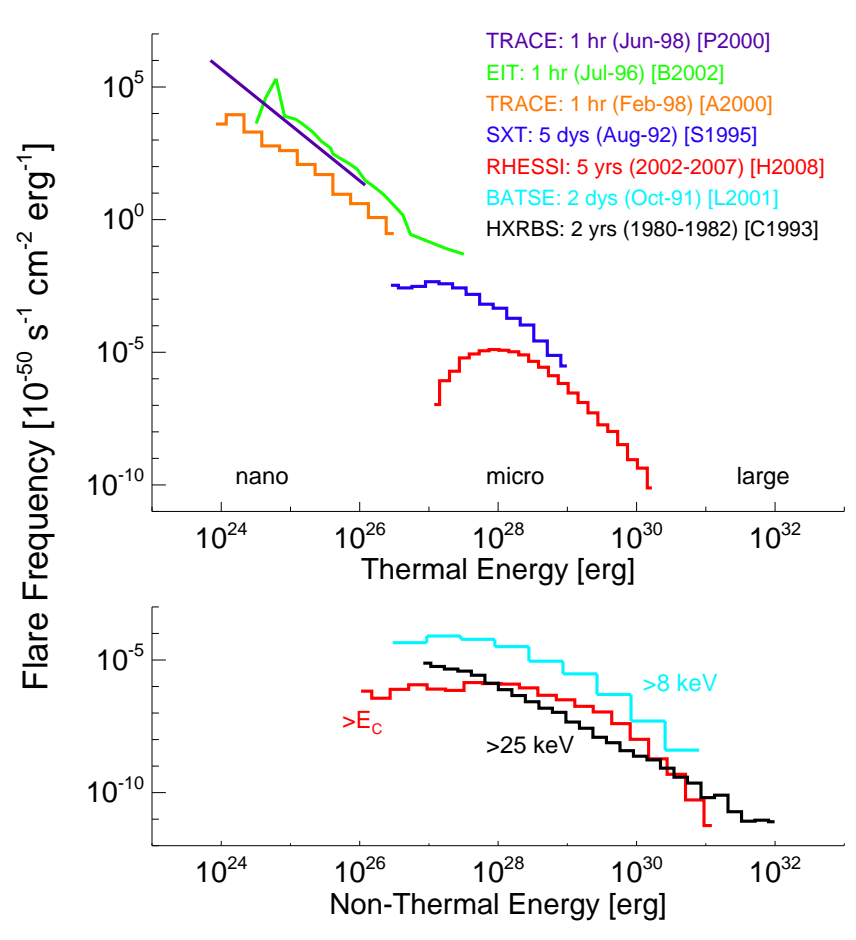

Fig. 2.1 The energy distributions for solar flares. The nonthermal energy distribution is shown for large flares $>25 \mathrm{keV}$ observed with $S M M / \mathrm{HXRBS}$ (Crosby et al. 1993), microflares $>8 \mathrm{keV}$ from CGRO/BATSE Lin et al. 2001a) and microflares $>E_{\mathrm{C}}$ (above the low energy cutoff) from RHESSI (Hannah et al. 2008a). The thermal energy distribution is shown for microflares with RHESSI Hannah et al. 2008a) and Yohkoh/SXT (Shimizu 1995) and EUV nanoflares with TRACE (Parnell \& Jupp 2000; Aschwanden et al. 2000) and SOHO/EIT (Benz \& Krucker 2002). This figure is deceptive as it is comparing energy distributions of different flare energy components, each involving different instrument and selection effects, and were obtained over different periods of different solar cycles.

Table 2.1 Different flare classifications and the associated ranges of GOES flux (SXRs) and HXRs.

\begin{tabular}{|c|c|c|c|c|c|}
\hline Flare Size & Description & $\begin{array}{l}\text { Energy } \\
\text { [erg] }\end{array}$ & $\begin{array}{l}\text { GOES } \\
\text { Class }\end{array}$ & $\begin{array}{l}\text { GOES Flux } \\
{\left[\mathrm{W} \mathrm{m}^{-2}\right]}\end{array}$ & HXR emission \\
\hline Large $\backslash$ normal & Active region phenomena & $\leq 10^{33}$ & $\mathrm{C}, \mathrm{M}, \mathrm{X}$ & $10^{-6}-10^{-3}$ & $>25 \mathrm{keV}$ \\
\hline Micro $\backslash$ ARTB $^{1}$ & Active region phenomena & $\sim 10^{27}$ & $\mathrm{~A}, \mathrm{~B}$ & $10^{-8}-10^{-6}$ & $10-30 \mathrm{keV}$ \\
\hline Nano & $\begin{array}{l}\text { Unobserved basic unit of } \\
\text { localized impulsive energy } \\
\text { release or very small } \\
\text { (EUV) brightening }\end{array}$ & $\sim 10^{24}$ & $? \ll \mathrm{A}$ & $? \ll 10^{-8}$ & $?$ \\
\hline
\end{tabular}

1 “Active Region Transient Brightening” observed in SXRs (Shimizu 1995)

These two features can be seen in the GOES flaring rate shown in Figure 2.4 discussed in Section 2.2.1

Flare phenomena encompass all of the accessible wavelength ranges of electromagnetic radiation, as well as the emission of neutral particles, gaseous ejecta, and large-scale shock waves (e.g. Fletcher et al.2011). In the most energetic flares, all of these phenomena appear, considerably more intensely than in smaller flares and regardless of the detailed physics; this is the so-called big-flare syndrome (e.g., Kahler 1982). This makes these events helpful diagnostically. This property suggests that if a particular phenomenon (e.g., white-light continuum) is not observed in a weaker flare (as not distinguishable from the background), the process may still function in the same way. Non-scalable properties might exist; one 


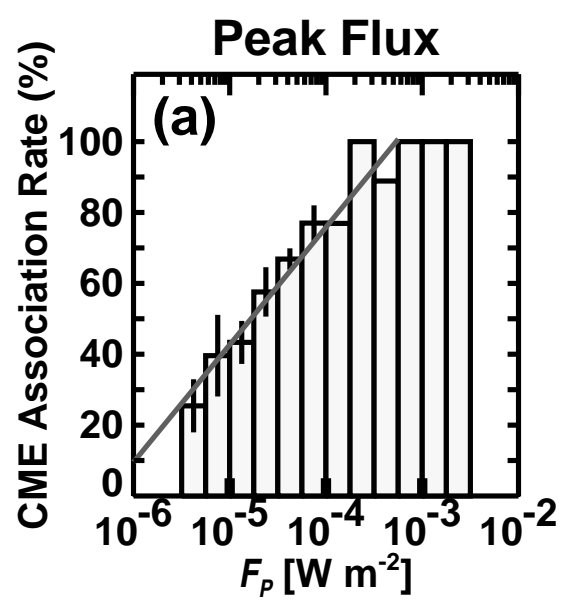

Fig. 2.2 The association rate of CMEs with flares as a function of the flare peak GOES flux (Yashiro et al. 2006). Only flares from C-class $\left(10^{-6} \mathrm{~W} \mathrm{~m}^{-2}\right)$ to $\mathrm{X}$-class $\left(>10^{-4} \mathrm{~W} \mathrm{~m}^{-2}\right)$ are included in this survey. Reproduced by permission of the AAS.

that is often cited is the maximum energy attained by accelerated particles. An emission such as $\pi^{0}$-decay $\gamma$-ray emission might thus have a non-linear threshold dependence; see Vilmer et al. (2010). Of course, given that there weak flares are considerably more numerous, exceptional cases can often be found, such as microflares with nonthermal emission to remarkably high energies (e.g., Hannah et al. 2008b).

\subsubsection{Flare relationship to CMEs and SEPS}

There has been a long debate whether ejection of material from the corona, namely a Coronal Mass Ejection (CME), triggers a flare, or vice-versa, but it is now generally accepted that they are both consequences of coronal energy release through the reconfiguration of the magnetic field. The issue of whether a flare or CME is produced will depend on the magnetic field configuration (Wang \& Zhang 2007). Using a large survey of nearly 7000 CMEs, Yashiro et al. (2004) found that all the most energetic (>X2) flares have CMEs; see Figure 2.2 (Yashiro et al. 2006). The largest and fastest CMEs are generally associated with such large flares. The rate for C-class flares is about $20 \%$ and for M-class about $40 \%$. Of course, there may be a sensitivity issue which limits the ability to observe the small CMEs associated with microflares. Studies of flares that do not have associated CMEs have found these events to have a correlation between peak intensity and flare duration (Kay et al.2003). No such correlation was found for CME flare events, suggesting a physical difference in the time development of a flare when a CME is involved. CMEs appear to have broadly the same range of characteristics whether or not they are associated with a flare, but the fastest and broadest CMEs are always associated with the most energetic flares (Vršnak et al. 2005). In terms of the energy partition between flares and CMEs, the CME kinetic energy ranges may be comparable to the total flare energy (Emslie et al. 2005).

The ejection of highly accelerated (up to several MeV) electrons, protons and heavy ions from the Sun into interplanetary space, called Solar Energetic Particles (SEPs), are sometimes associated with flares (e.g. Reames 1999). Impulsive SEP events demonstrate a rapid burst of electron enhancement often related to the impulsive HXR phase of flares, suggesting that the same coronal energy release is responsible for the flare and SEPs. These energetic electrons therefore provide an important diagnostic of flare particle acceleration, especially when combined with HXR flare observations. Type III radio bursts are also produced by en- 

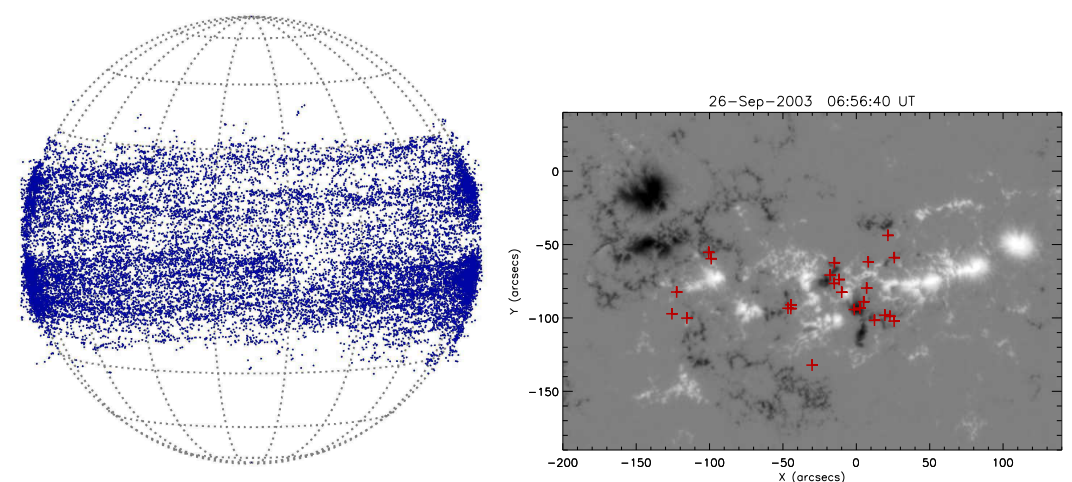

Fig. 2.3 (Left) The locations of 24,097 microflares observed with RHESSI between March 2002 and March 2007 Christe et al. 2008a). All of the confirmed events could be associated with active regions. Reproduced by permission of the AAS. (Right) The locations of one day of RHESSI microflares from active region 10456 (Stoiser et al. 2007). Of 53 events found, 24 could be imaged. The centroid positions (3-6 keV) are indicated by crosses and overplotted on the MDI (Michelson Doppler Imager of $\mathrm{SOHO}$ ) magnetogram.

ergetic electrons escaping from the Sun into interplanetary space, see Section 2.2.3. Gradual SEP events show a slower enhancement of energetic protons, thought to be accelerated by a CME-associated shock in interplanetary space rather than in the coronal energy release. Although these proton events are not directly related to flares, Kiplinger (1995) showed that flares that were associated with $10 \mathrm{MeV}$ proton events predominantly demonstrated progressive hardening of the HXR photon spectra into the decay phase of the HXR emission. In contrast, the other population of HXR events not associated with SEPs exhibit softening spectra in the decay phase. A similar result was found for X-class flares during the January 2005 solar storm event (Saldanha et al. 2008). In these flares, four out five showed spectral hardening with RHESSI and were associated with interplanetary proton events. A study of 37 events with RHESSI found that the majority of events that demonstrated HXR spectral flattening (12 out of 18) produced SEPs (detected with GOES $>10 \mathrm{MeV}$ proton data and WIND/3DP $0.1-1 \mathrm{MeV}$ proton and $30-500 \mathrm{keV}$ electron data) and all without flattening (19 flares) did not produce SEPs (Grayson et al. 2009).

\subsection{Microflares}

\subsubsection{Association with active regions}

"Subflares" had always been known to the H $\alpha$ observers (e.g., Smith \& Smith 1963) as events that were small in area but not necessarily faint. The term "microflare" was introduced in the 1980s by Schadee et al. (1983) for SXRs, and by Lin et al. (1984) for HXRs. The presence of X-rays, the occurrence of subflares with "brilliant" $\mathrm{H} \alpha$ classification (high intensity), and the discovery of faint microwave bursts (Gopalswamy et al. 1994; Gary et al. 1997) in association with SXR microflares made it clear that the basic flare physics extended over a wide magnitude range. Flares generally (including subflares and microflares) show both thermal and nonthermal emission, indicating the presence of particle acceleration and plasma heating. In terms of GOES classification these events are typically A- and B-class events, down to and beyond the sensitivity limit of the GOES detectors. 

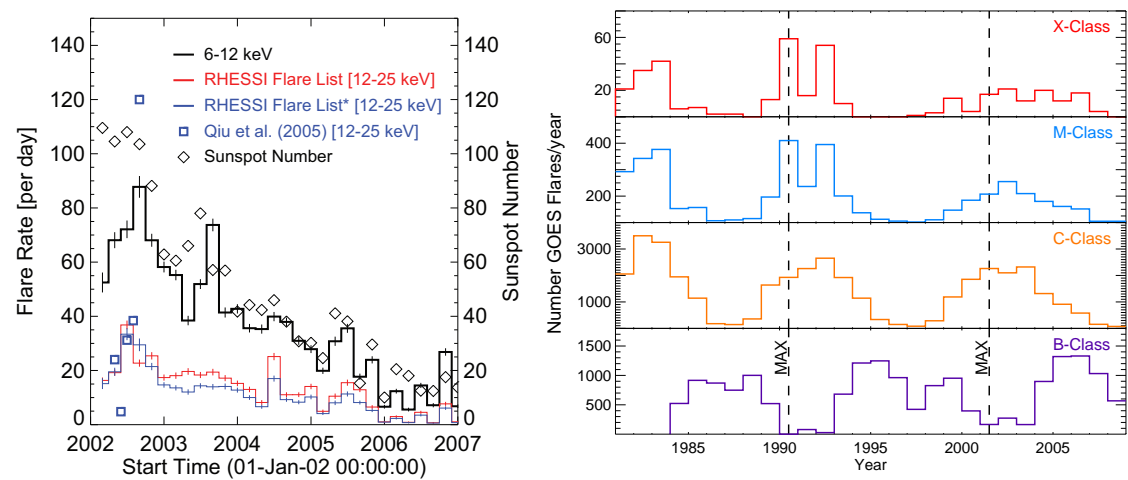

Fig. 2.4 (Left) Occurrence rate of RHESSI microflares ( $<\mathrm{C} 1$ events) Christe et al. 2008a), showing a clear decline over the waning years of Cycle 23. Reproduced by permission of the AAS. (Right) The number of GOES flares per year per flare class for X-class (top) through M, C to B-class (bottom). The microflares seem to be anti-correlated with the larger flares, which would be expected from the increased background during times of high activity. Note that B-class flare data was only available from 1984 (data from Milligan 2009).

The HXR observations prior to RHESSI normally suffered from poor sensitivity due to lack of access to lower hard X-ray energies $(<15 \mathrm{keV})$, hence these observations missed most of the microflares. The problem was the use of thick fixed attenuators to reduce the excessive low energy counts in large flares. One exception to this was the HXIS1 imager on the Solar Maximum Mission (SMM) (Schadee et al. 1983), which covered the $3-30 \mathrm{keV}$ range with a restricted field of view and very small detectors, thus avoiding saturation. Because of this, the main HXIS results were in a spectral region comparable with the GOES data. At lower energies $(0.25-4 \mathrm{keV})$, the Soft X-ray Telescope (SXT) on Yohkoh found microflares to occur in active regions (Shimizu 1995).

The first observation of HXR microflares was with balloon-borne detectors that could observe only down to about $15 \mathrm{keV}$ due to atmospheric absorption, and which had high sensitivity from the use of large-area, low-background detectors (Lin et al. 1984). Subsequently with Yohkoh/HXT, emission at $14-23 \mathrm{keV}$ was found to be associated with SXR brightenings in active regions (Nitta 1997). Microflares were also detected at $8-13 \mathrm{keV}$ with CGRO/BATSE (Lin et al. 2001b). However, it was not until RHESSI that the detailed associations of these HXR microflares could be readily investigated.

RHESSI's introduction of a movable attenuating shutter system (Lin et al. 2002) allowed the attenuators only to be deployed when the detectors were saturated. With no shutters, the detectors become saturated once solar emission (either background emission from active regions or flares) is approximately greater than GOES C1 level. RHESSI microflares are therefore flares that occur when the attenuating shutters are out; they are typically sub-C-class events, $<10^{-6} \mathrm{~W} \mathrm{~m}^{-2}$ in the $1-8 \AA$ GOES band. The full view and sensitivity of RHESSI is available to such events, providing the first comprehensive HXR view of microflares, with both imaging and spectroscopy. In addition to this, RHESSI introduced Ge detectors with resolution much superior to the scintillation counters previously used for HXR observations, allowing the properties of steep spectra to be accurately determined.

RHESSI microflares appear to be exclusively localized to active regions. Figure 2.3 (left panel) shows this explicitly with the positions of 24,097 events, all of which are identified

\footnotetext{
1 Hard X-ray Imaging Spectrometer.
} 
as coming from active regions (Christe et al. 2008a). These events were identified from analyzing all count-rate spikes in the RHESSI 6-12 keV energy range between March 2002 and March 2007, at times when the attenuating shutters were out. A total of 25,705 events were found, though only 24,097 events could be imaged to give positions on the solar disk. The events not counted include some of the faintest true microflares, but are predominantly misidentified particle events (which gave a non-solar disk position) or events close to the rotation axis of RHESSI (events close to where the spacecraft rotation axis is pointing will have no modulation in their detected signal and hence no spatial information can be recovered). There is repeated microflaring from active regions, thus tracing out the location of a region as it moves across the disk. The microflaring rate varies greatly between active regions. Detailed RHESSI imaging of all the microflares from one active region compared to MDI magnetograms shows that the microflare emission occurred at multiple locations throughout the active region (Stoiser et al. 2007), though many originated in one localized area of the region (Figure 2.3 right panel). Another sample of microflares showed that they mostly occur near magnetic neutral lines in active regions (Liu et al. 2004).

As expected, then, Figure 2.4 (left panel) shows that these events tend to follow the solar cycle in their occurrence frequency. We note that it is difficult to follow the microflare rate over a whole solar cycle because the solar X-ray background level also follows the solar cycle. Fewer microflares are observed during periods of high solar activity as they are hidden by the high background from active regions. This can be clearly seen in the number of GOES B-class flares (right panel of Figure 2.4. Their rate appears unexpectedly to be almost anticorrelated with the solar cycle. This observational bias means that the microflaring rate is only accurately known during times of lower activity/solar background.

As the HXR microflares observed by RHESSI are inherently active-region phenomena, it seems entirely reasonable to associate them with microflares (Schadee et al. 1983) and the active-region transient brightenings (ARTBs) seen in soft X-rays (Shimizu 1995). This had been previously postulated (Tsuneta \& Lemen 1993) and early evidence suggested it to be correct (Nitta 1997), but the confirmation was only possible with RHESSI's hard X-ray sensitivity.

\subsubsection{Physical properties}

The X-ray time profiles of typical microflares (examples given in Figure 2.5) demonstrate a similar structure to those in typical large flares: a short impulsive burst at higher energies (HXRs) followed by a more gradual thermal emission at lower energies (SXRs). Microflares also demonstrate other temporal phenomena seen in large flares like more gradual emission or pre-impulsive behavior though the timescales are generally shorter (seconds to minutes instead of several to tens of minutes).

The first HXR spectra of microflares (top panel of Figure 2.6 showed only a power-law component, characteristic of nonthermal emission, due to the detector being sensitive only above about $13 \mathrm{keV}$ (Lin et al. 1984). RHESSI spectra (bottom row of Figure 2.6) can be obtained with moderate energy resolution down to a few $\mathrm{keV}$. This allows the thermal component, and the transition from it to the nonthermal component, to be seen. These spectra (from the time of peak emission just after the impulsive phase) are similar to those seen in more energetic flares, except that (a) the thermal emission tends to be at lower temperatures and (b) the nonthermal component tends to have a steeper spectrum (fewer electrons accelerated to high energies). In the bottom left panel of Figure 2.6 we have a typical RHESSI microflare with a fitted temperature of about 13 MK. The Fe feature (e.g., Phillips 2004) is clearly in evidence at about 6-7 $\mathrm{keV}$ and a nonthermal spectrum above $8 \mathrm{keV}$ with an 

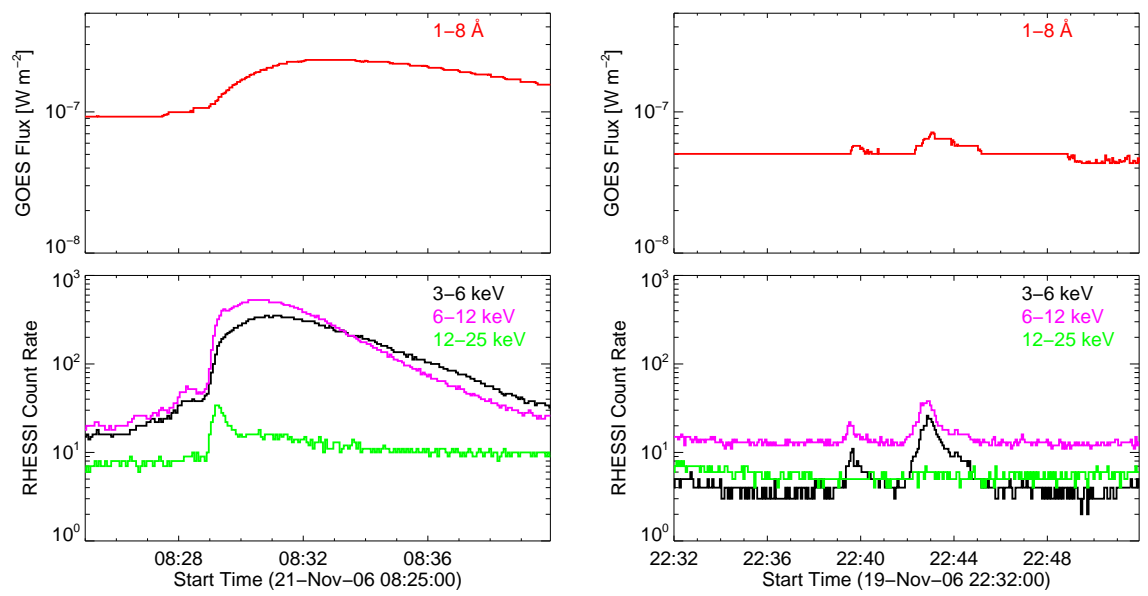

Fig. 2.5 The GOES $1-8 \AA$ Aightcurve (top panels) and RHESSI lightcurves for some example microflares. (Left:) A single B-class microflare (B1 with pre-flare background subtracted) which shows the classic flare time profile: impulsive at higher energies with a slower rise and more gradual fall at lower energies. This event also shows some pre-flare emission. (Right:) two A-class events (A1 and A3, background-subtracted) both of which have no emission above $12 \mathrm{keV}$ observed by RHESSI; their nonthermal emission hidden by the background. Note that the background level in these microflares is predominantly solar for GOES, but terrestrial and instrumental in origin for RHESSI.

$\mathrm{E}^{-5.15}$ power law. The other RHESSI spectrum shown (bottom right panel of Figure 2.6) is of an anomalously hard microflare, which despite being a GOES A2 event (near the limit of the GOES sensitivity) demonstrates strong nonthermal emission to relatively high energies, just as in much more powerful events (Hannah et al. 2008b). One should note, however, that the early microflare HXR spectra of Lin et al. (1984) were more sensitive above about 30 $\mathrm{keV}$ since their shielded detectors were able to reduce the background clearly evident in the RHESSI spectra. These Lin et al. (1984) microflares also demonstrate steep nonthermal emission of $I(\varepsilon) \propto \varepsilon^{-\gamma}$ with $\gamma=4$ to 6 that continue to energies normally obscured by background in RHESSI observations.

RHESSI microflares are not spatially small and typically show an elongated loop-like structure, with higher-energy HXR emission from the footpoints at the ends of the loop (e.g., Krucker et al. 2002; Liu et al. 2004; Stoiser et al. 2007; Hannah et al. 2008b). Figure 2.7 shows an example of this overlaid on an EUV image from TRACE. We interpret the EUV loop as the cooling post-flare loop which was first seen at higher temperatures in the RHESSI image. This hot material could be driven (evaporated) from the chromosphere by the energy deposition of the nonthermal electrons penetrating to the loop footpoints (Fisher et al. 1985; Abbett \& Hawlev 1999; Brosius \& Phillips 2004) and producing HXR sources there. Several RHESSI microflares have also been observed with the HXR loop connecting two bright $\mathrm{H} \alpha$ kernels (Liu et al. 2004), another possible signature of the energy deposition of nonthermal electrons in the lower atmosphere. Previous work had shown the association between microflare SXR and $\mathrm{H} \alpha$ emissions (Shimizu et al. 2002), consistent with the relationship known from ordinary flares (Thomas \& Teske 1971).

Figure 2.8 shows the fitted lengths and widths of the thermal emission (4-8 keV) for 16 seconds about the time of peak emission in 6-12 keV for almost 19,000 RHESSI microflares (Hannah et al. 2008a). These parameters are from simple forward-fitted image models and 

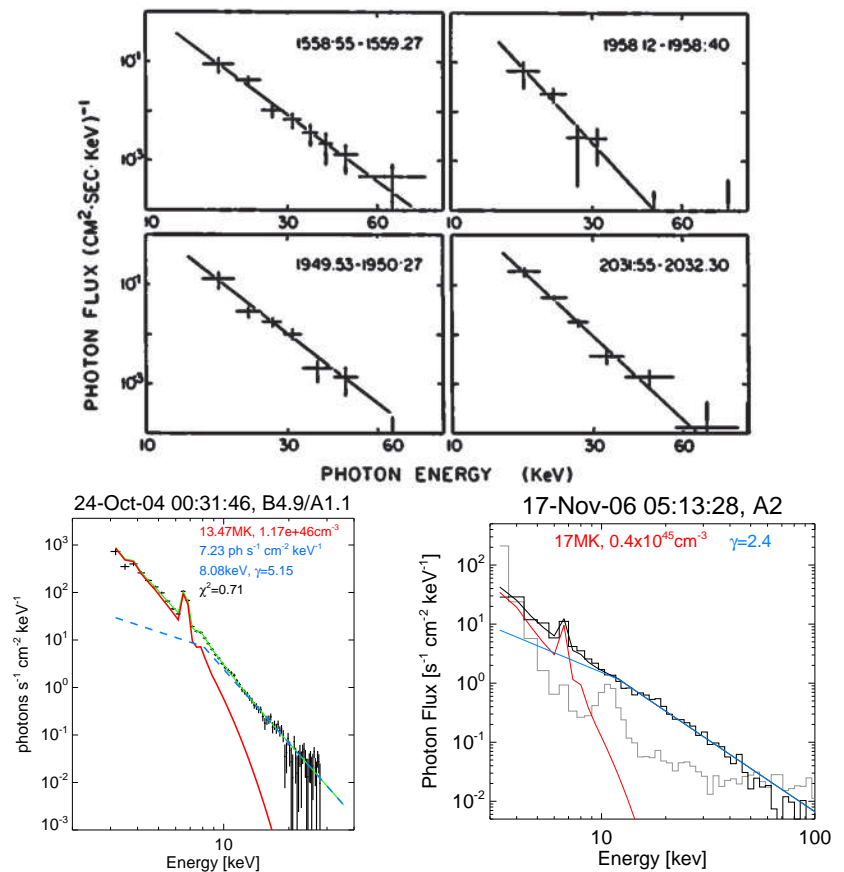

Fig. 2.6 (Top four panels:) the first HXR microflare spectra from a balloon-borne instrument (Lin et al. 1984), and (bottom two panels: spectra from RHESSI showing a typical microflare Hannah et al. 2008a) (left) and one with a particularly hard spectrum (right) (Hannah et al. 2008b). The red and blue lines are the thermal and nonthermal components of the model, respectively. In the RHESSI spectra, the upper limit of the nonthermal component is hidden by the instrumental background (the large errors bars in the bottom-left spectrum and the grey histogram in the bottom-right spectrum). Reproduced by permission of the AAS.
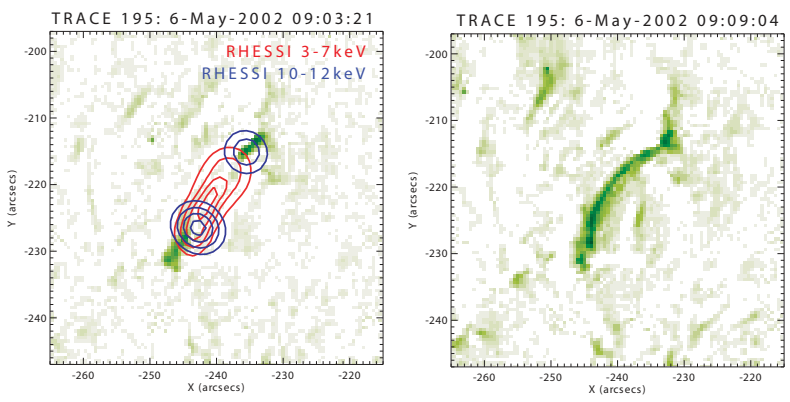

Fig. 2.7 Overlay of RHESSI thermal (red) and nonthermal (blue) contours on TRACE, showing the typical flare and microflare scenario of (left) footpoints depositing their energy to heat chromospheric material that expands upwards, filling the loops connecting them. (Right:) his material then cools, emitting brightly in EUV. Updated version of figure from Krucker et al. (2002).

show that the microflares have a clear tendency to be elongated, as we would expect from their identification with the loops that characterize SXR microflares (e.g., Shimizu 1995). The distribution of the loop length has a peak well above RHESSI's angular resolution, so we conclude that these microflares may not be inherently small-scale. Many ordinary flares, though more energetic, have dimensions in this range. In addition, these lengths are not correlated with the microflare peak GOES flux or peak RHESSI emission (Hannah et al. 2008a) 


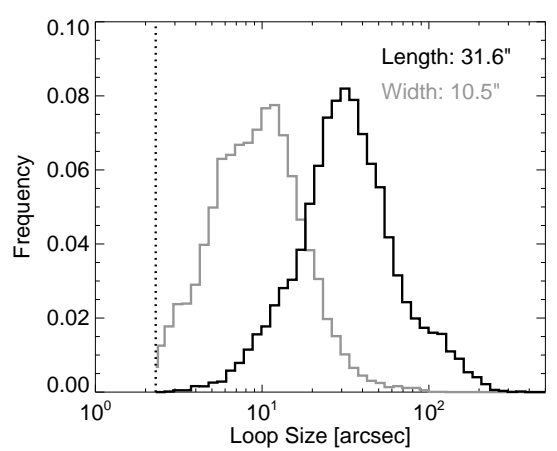

Fig. 2.8 Loop arc length and width distributions for 18,656 microflares imaged by RHESSI indicating that they are predominantly elongated structures (Hannah et al. 2008a). Thus microflares are not necessarily spatially small. Reproduced by permission of the AAS.

\subsubsection{Associated ejecta and radio emission}

Radio signatures generally guide us to the corona, since the opacity (and the minimum height of formation of the emission) increases in height almost monotonically with wavelength (e.g., White et al. 2010). Millimeter-centimeter wavelengths (microwave GHz emission) originate mainly in the lower atmosphere, meter-waves (100s $\mathrm{MHz}$ to $\mathrm{MHz}$ radio emission) in the lower corona, and the longest wavelengths right out into the solar wind. The longer-wavelength radio observations thus often show ejecta, especially via nonthermal plasma signatures (e.g., Pick \& Vilmer 2008).

The microflare gyrosynchrotron emission is closely correlated in time to the HXR emission (Qiu et al. 2004; Kundu et al. 2006), indicating that they are both signatures of the accelerated flare electrons. The previous observations of SXR microflares also demonstrated a correlation with microwave emission (Gary et al. 1997; Nindos et al. 1999). The microwave spectral index for microflares is found to be flatter (harder) than the RHESSI HXR spectra (Qiu et al. 2004). This is thought to be due to the microwave emission originating from electrons with higher energies than those producing X-rays (Nitta \& Kosugi 1986). This discrepancy in spectral indices is also observed in larger flares (Silva et al. 2000) though it is possibly greater in microflares. This may be due to the HXR spectra being typically steeper in microflares than large flares. A B-class microflare was observed to have nonthermal microwave emission from MeV-energy electrons but showed no HXRs at lower energies (Raulin et al. 1999). This behavior could result from trapping of the lower-energy electrons during the peak of the microwave emission from the higher-energy electrons.

Imaged microwave emission using the Nobeyama radioheliograph (NoRH) for RHESSI microflares indicated similarly sized and separated footpoints in both HXR and microwave emission (Kundu et al. 2006); see Figure 2.9 The higher-frequency microwaves come from the footpoints, and the lower-frequency from the connecting loop (Kundu et al. 2005, 2006).

RHESSI microflares are often associated with Type III radio bursts, produced by electron beams escaping from the corona and often subsequently detected as SEPs. These are the "fast drift" bursts identified with weakly relativistic electron beams (e.g., Wild et al. 1963). The HXR emission in these cases still seems to be a signature of accelerated electrons reaching the lower atmosphere at loop footpoints, although the radio emission comes from accelerated electrons of similar energies moving outwards from a postulated coronal acceleration site. An example of six microflares identified with RHESSI and their associated $\mathrm{MHz}$ radio emission observed with WIND/WAVES is shown in Figure 2.10. Here we can see the time correlation of the HXR emission and the high-frequency radio emission. The mi- 

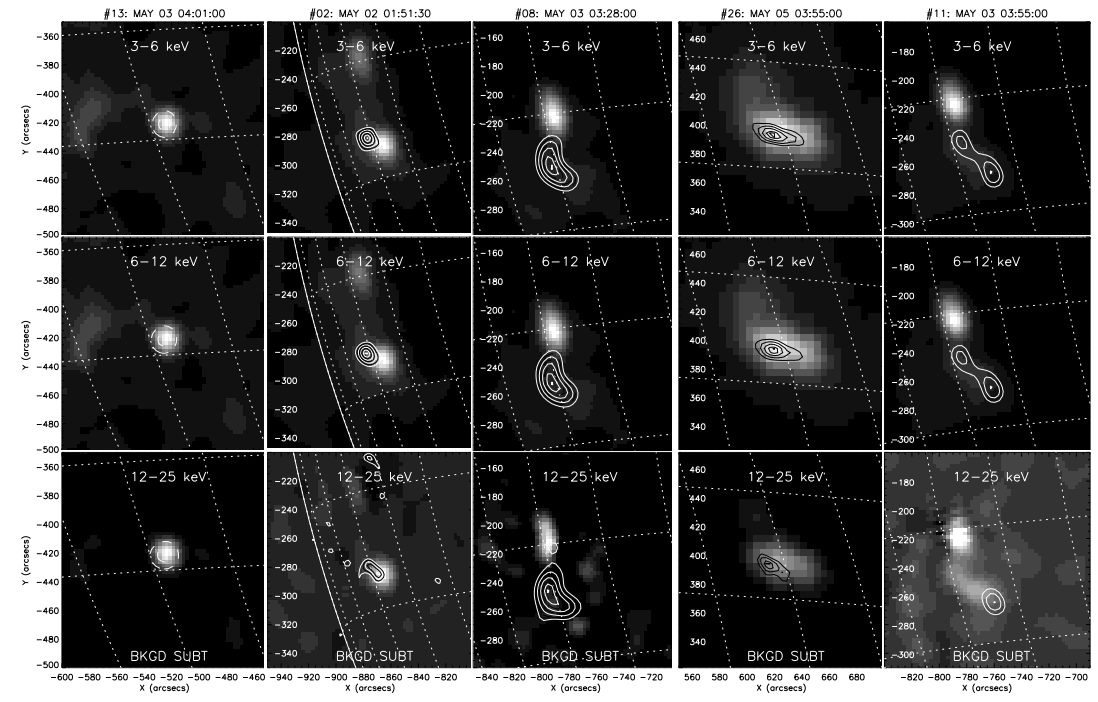

Fig. 2.9 Five microflares (one per column) imaged in different HXR energy bands (increasing from top row to bottom) with RHESSI (contours), overplotted on NoRH $17 \mathrm{GHz}$ images (Kundu et al. 2006). The bottom microwave image is background-subtracted. Similar-sized footpoints and loop structures are seen in both HXRs and microwaves.

croflare with the largest HXR flux produced the brightest radio burst. The association with Type III bursts establishes that electrons accelerated in the microflares have access to open field lines (or field lines that extend into the upper corona), not requiring an eruption prior to the event. An association between Type III bursts and small SXR flares had previously been found by Fuerst et al. (1982).

The presence of open field lines in microflares has also been seen with the association of jets of material flowing out of the flare region (Shimojo et al. 1996). For RHESSI HXR microflares, such jets have been observed in EUV with TRACE (Christe et al. 2008b) and in EUV and SXRs with Hinode (Chifor et al. 2008). In the former case, multiple microflares and type III burts were observed, occurring every few minutes, with EUV jets associated with the largest microflares (Christe et al. 2008b). In the latter case, the recurring jets occurred on a timescale of hours and were attributed to chromospheric evaporation flows due to recurring coronal magnetic reconnection (Chifor et al. 2008).

This association with jets is also well observed in soft X-rays (Strong et al. 1992; Shibata et al. 1992). Indeed all such jets, including those in the quiet Sun, appear to have loop brightenings at their base. A jet appears as a collimated flow, implying the prior existence of large-scale or even open magnetic fields. Type III radio bursts, known to be produced by electron beams escaping from the corona into the solar wind, have a strong association with the soft $\mathrm{X}$ ray jets as well (Aurass et al. 1994; Kundu et al. 1995). Thus the microflare/jet events also have thermal and nonthermal attributes just as major flares do, but the jet is relatively more prominent in the microflare domain. 


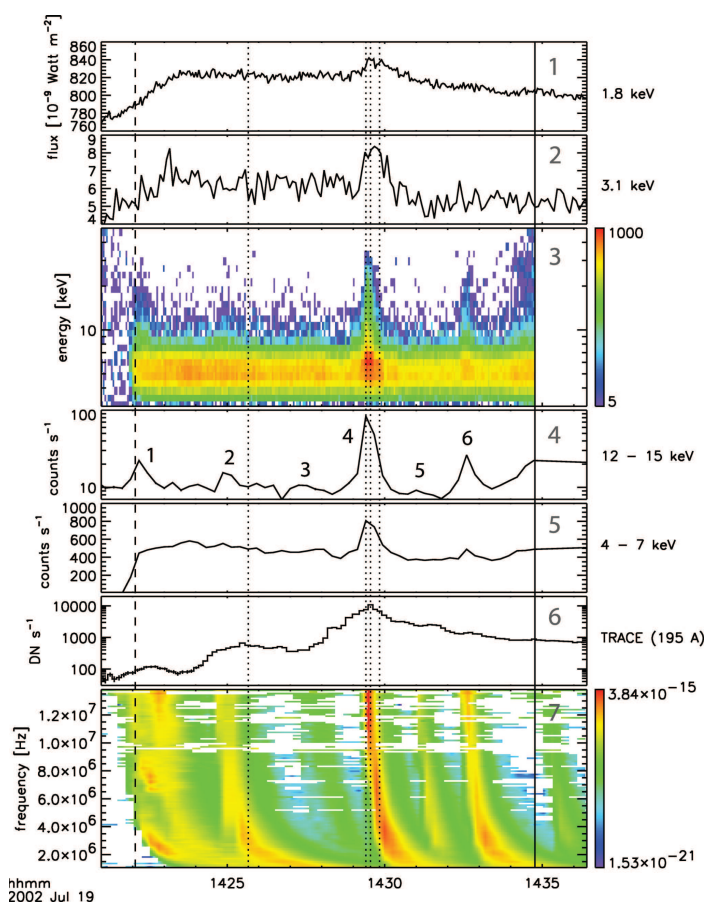

Fig. 2.10 The lightcurves and radio spectrograms of six RHESSI microflares from July 19, 2002 (Christe et al. 2008b). The top two panels show the GOES lightcurves in $1-8 \AA$ (with a B8 background level) and $0.5-4 \AA$. The next three panels show the RHESSI spectrogram with night-time background removed and lightcurves in $12-15 \mathrm{keV}$ (nonthermal) and $4-7 \mathrm{keV}$ (thermal). The EUV lightcurve from TRACE and a radio spectrogram from WIND/WAVES are shown in the bottom two panels. The radio emission starts at around $14 \mathrm{MHz}$ and drifts down to $2 \mathrm{MHz}$. It is well correlated with the RHESSI HXR lightcurve. Reproduced by permission of the AAS.

\subsection{Nanoflares and non-active-region phenomena}

We have seen above that the RHESSI microflares occur only in active regions and have a strong kinship with "ordinary" flares. How do these relate to X-ray bright points Golub et al. 1974), network flares (Krucker et al. 1997), plumes (Ahmad \& Webb 1978), or any of many more types of weak flare-like brightenings seen both inside and outside the active regions? Note that EUV observations such as those of SOHO/EIT or TRACE may be much more sensitive than SXR (or HXR) observations of transient features at ordinary coronal temperatures of order 1 MK (Porter et al. 1987; Krucker \& Benz 1998). Many of these phenomena have been related to Parker's nanoflares and to the problem of coronal heating. The nanoflare description of these EUV events is justified, in the sense that an EUV brightening may have a much smaller event energy than a microflare observed in SXRs, HXRs, or microwaves. However it is often difficult to place these different sorts of observations on a uniform energy scale. We deal with this at length in Section 3.1 below.

Outside of active regions, the most prevalent X-ray features of SXR images are the Xray bright points (XBPs), originally discovered with rocket-borne X-ray imagers in the late 1960s and studied statistically with data from the X-ray telescope on Skylab (Golub et al. 1974).

Subsequent observations had sufficient resolution to detect hot (about $2 \mathrm{MK}$ ) loop-like (about 10" in length) properties and footpoints in the XBPs (Moses et al. 1994; Strong et al. 1992; Kotoku et al. 2007), pointing to a possible relationship with flare physics. Typically hundreds of XBPs are visible, uniformly spread across the solar disk (Golub et al. 1974), their number changing little once observational bias, due to the dominant active region emission, is removed (Hara \& Nakakubo-Morimoto 2003). This spatially-uniform and time- 

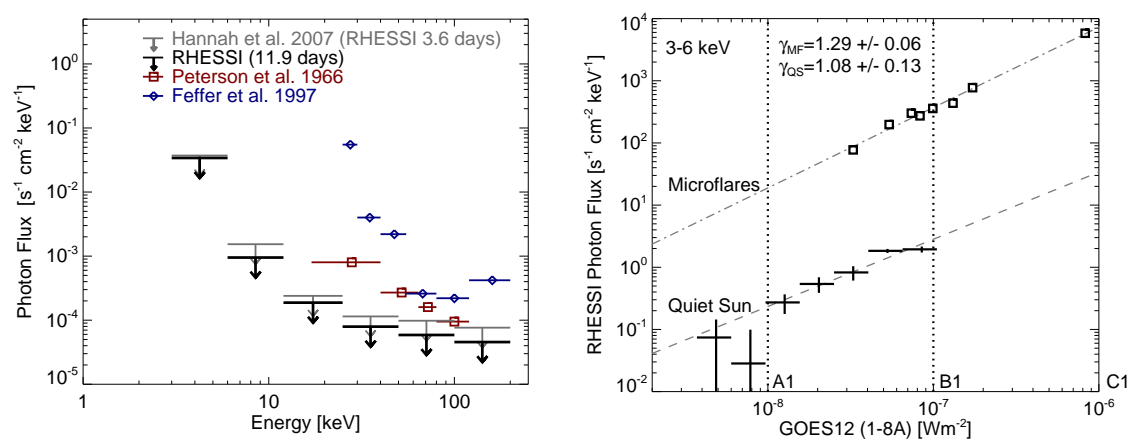

Fig. 2.11 Observations and upper limits of the quiet-Sun hard X-ray flux found with RHESSI. (Left:) the RHESSI upper limits to the quiet Sun emission at $3-200 \mathrm{keV}$ in comparison to previous observations (Hannah et al. 2010), including the first RHESSI quiet Sun limits (Hannah et al. 2007b). (Right:) comparison of the 3-6 keV flux observed with RHESSI to the $1-8 \AA$ GOES emission for the quiet Sun and offpointed microflares. The $\gamma_{\mathrm{MF}}$ and $\gamma_{\mathrm{QS}}$ indicate the index of power-law fits to the data, the dashed and dashed-dotted lines (Hannah et al. 2007b). Reproduced by permission of the AAS.

invariant distribution across the solar surface is expected as the XBPs are associated with the magnetic fields related to surface convective flows (the granulation and supergranulation of the quiet photosphere) rather than with the active-region fields responsible for sunspot fields. X-ray bright points are associated with connectivity changes in the network magnetic field. The underlying fundamental physical processes could be the same as for active-region microflares. Often, XBPs may persist for hours to days, unlike the transient active region flares, and thus their behavior may resemble that of quiescent active-region loops (e.g., Yoshida \& Tsuneta 1996). In the absence of solar active regions, the XBPs and the corona of the quiet Sun provide a basal X-ray emission level. Jets are sometimes observed from XBPs, particularly in polar regions (Shibata et al. 1992; Shimojo et al. 2007; Cirtain et al. 2007; (Savcheva et al. 2007) where there is easy access to open field lines.

There have been no HXR observations of XBPs. Such emission therefore must be below the sensitivity limits of current spacecraft, which have been optimized to observe brighter active-region flares. Radio emission associated with XBPs has been observed, but in many instances it is consistent with thermal instead of nonthermal emission (Nitta et al. 1992). Network flares, associated with the magnetic network boundaries, are about an order of magnitude fainter than XBPs and are more transient, lasting for only about 10 minutes (Krucker et al. 1997). Radio emission has also been observed in these events, but again in the majority of the cases it is consistent with thermal emission, and in only a few cases could it be associated with nonthermal gyrosynchrotron emission from accelerated electrons.

Determining whether there is nonthermal emission from a population of accelerated electrons outside of active regions and in the quiet Sun would provide important insights into the nature of possible small-scale steady-state energization processes in the solar corona. Although RHESSI has unprecedented sensitivity over $3-25 \mathrm{keV}$, which has greatly aided the study of small active-region flares (see Section 2.2, , emission outside active regions still remains elusive.

Investigating the non-flaring properties of active regions (McTiernan 2009) or the quiet Sun with RHESSI is a non-trivial task. RHESSI's imaging method is designed for bright compact flares and ill-suited for the spatially-diffuse emission expected from coronal heating. Instead, upper limits to the quiet Sun HXR flux can be obtain by offpointing RHESSI 
Table 2.2 Table of the power-law indices $\alpha$ found from statistical surveys of the fluxes of X-ray flares. The top set are predominantly SXR surveys, the bottom HXR surveys.

\begin{tabular}{|c|c|c|c|c|c|}
\hline Index $\alpha$ & Energy Range & Duration & Number & Quantity & Instrument \& Reference \\
\hline 1.86 & $7.7-12.5 \mathrm{keV}$ & 1967(2 weeks) & 177 & Peak & OSO-3 (Hudson et al. 1969) \\
\hline 1.75 & $1-6.2 \mathrm{keV}^{1}$ & 1966-1968 & 4028 & Peak & Explorer-33/35 (Drake 1971a) \\
\hline 1.44 & $1-6.2 \mathrm{keV}^{1}$ & $1966-1968$ & 4028 & Fluence & Explorer-33/35 (Drake 1971a) \\
\hline 1.79 & CA XIX & 1980-1989 & & Peak & BCS (Lee et al. 1995) \\
\hline 1.86 & $1.5-12.4 \mathrm{keV}^{2}$ & 1980-1989 & & Peak & GOES (Lee et al. 1995) \\
\hline 1.88 & $1.5-12.4 \mathrm{keV}^{2}$ & 1993-1995 & 1054 & Peak & GOES (Feldman et al. 1997) \\
\hline 2.11 & $1.5-12.4 \mathrm{keV}^{2}$ & $1976-2000$ & 49409 & Peak & GOES (Veronig et al. 2002a) \\
\hline 2.03 & $1.5-12.4 \mathrm{keV}^{2}$ & $1976-2000$ & 49409 & Fluence & GOES (Veronig et al. 2002a) \\
\hline 1.8 & $20 \mathrm{keV}$ & 1971-1972 & 123 & Peak $^{\text {ph }}$ & OSO-7 (Datlowe et al. 1974) \\
\hline 2.0 & $20 \mathrm{keV}$ & $27-06-1980$ & 25 & Peak $^{\text {ph }}$ & Balloon (Lin et al. 1984) \\
\hline 1.8 & $>30 \mathrm{keV}$ & $1980-1985$ & $>7000$ & Peak & HXRBS (Dennis 1985) \\
\hline $1.66-1.75$ & $>25 \mathrm{keV}$ & 1980-1989 & $>7000$ & Peak & BATSE (Schwartz et al. 1992) \\
\hline 1.61 & $>25 \mathrm{keV}$ & 1991 & 1262 & Peak & HXRBS (Schwartz et al. 1992) \\
\hline 1.75 & $>26 \mathrm{keV}$ & $1978-1986$ & 4356 & Peak & ICE (Lee et al. 1993) \\
\hline $1.70-1.86$ & $>30 \mathrm{keV}$ & $1980-1984$ & 3578 & Peak & HXRBS (Bai 1993) \\
\hline 1.73 & $>25 \mathrm{keV}$ & 1980-1989 & 7045 & Peak & HXRBS (Crosby et al. 1993) \\
\hline 1.59 & $>25 \mathrm{keV}$ & $1980-1989$ & 2878 & Peak $^{\text {ph }}$ & HXRBS (Crosby et al. 1993) \\
\hline $1.60-1.74$ & $>25 \mathrm{keV}$ & 1991-1994 & & Peak & CGRO (Biesecker 1994) \\
\hline $1.86-2.00$ & $>26 \mathrm{keV}$ & $1978-1986$ & 3468 & Peak $^{\text {ph }}$ & ICE (Bromund et al. 1995) \\
\hline 1.74 & $>60 \mathrm{keV}$ & 1980-1989 & 12327 & Peak & HXRBS (Kucera et al. 1997) \\
\hline 1.58 & $>10 \mathrm{keV}$ & 1989-1992 & 1551 & Peak & WATCH (Crosby et al. 1998) \\
\hline 1.56 & $>25 \mathrm{keV}$ & 1991-1994 & 5430 & Peak & BATSE (Aschwanden et al. 1998) \\
\hline 1.46 & $>50 \mathrm{keV}$ & 1991-1994 & 5430 & Peak & BATSE (Aschwanden et al. 1998) \\
\hline 1.50 & $3-6 \mathrm{keV}$ & $2002-2007$ & 24097 & Peak & RHESSI (Christe et al. 2008a) \\
\hline 1.51 & $6-12 \mathrm{keV}$ & $2002-2007$ & 24097 & Peak & RHESSI (Christe et al. 2008a) \\
\hline 1.58 & $12-25 \mathrm{keV}$ & $2002-2007$ & 24097 & Peak & RHESSI (Christe et al. 2008a) \\
\hline 1.71 & $4-8 \mathrm{keV}$ & $2002-2007$ & 18656 & Peak $^{\text {ph }}$ & RHESSI (Hannah et al. 2008a) \\
\hline
\end{tabular}

${ }^{1} 2-12 \AA,{ }^{2} 1-8 \AA$.

The default units for the hard X-ray results are count rate (observed flux) except those indicated by ${ }^{\mathrm{ph}}$ which are nominally instrument-independent photon fluxes.

from the Sun, so that the quiet solar signal is "chopped" as RHESSI rotates Hannah et al. 2007a). This process has obtained limits that are smaller and cover a wider energy range than previously found (Hannah et al. 2007b, 2010), as shown in Figure 2.11. A wide range of possible thermal and nonthermal emission is still consistent with these limits with only a hint as to the relationship between active region and non-active region flares. However, the nanoflare model for coronal heating, operating in a manner similar to active-region flares, does seem unlikely (Hannah et al. 2010). Future instruments with higher sensitivity and dynamic range could possibly find such HXR emission, which would be expected from a nanoflare heating model even in the absence of flare-like brightenings (e.g., Cargill \& Klimchuk 2004).

\section{Flare distributions \& parameter scaling}

The occurrence distribution function provides one good way to characterize a large number of observations. Many flare distribution functions have been published, and we can separate them into two classes: distributions of raw observable parameters (e.g., peak flux), and 
distributions of derived parameters (e.g., energy content). A derived parameter such as the total event energy might seem a more physically correct object, but the data manipulations required to get it will necessarily involve uncertain calibrations and "plausible" assumptions about the unobserved parts of the event. To make this latter point more concrete, consider the two GOES energy bands (0.5-4 $\AA$ and 1-8 $\AA$ ). These define an isothermal temperature and emission measure, but have little sensitivity at low coronal temperatures (e.g., 1-2 MK). Thus in principle a large emission measure and energy can be masked from view and go unaccounted for, systematically biasing the energy estimate to be a lower limit. In the following we provide a critical review of efforts to understand these different occurrence distribution patterns.

\subsection{X-ray flux distributions}

We summarize the reported X-ray flux and fluence (the time-integrated flux) distributions in Table 2.2 Most of the power-law indices $\alpha$ fall below the critical value of 2.0, meaning that the energetic events dominate, rather than the microflares (information on how to determine $\alpha$ accurately is detailed in Appendix A . An exception to this is the huge GOES event sample of Veronig et al. (2002a), which we showed in Figure 1.1 but in this case the authors deliberately did not attempt to correct for background counting rates. Indeed, this is a fundamentally ambiguous procedure, as noted by Bornmann (1990), and a typical source of systematic error. The SXR observations suffer more from this than the HXR observations because of their longer time scales. Determining the fluence also introduces bias: how does one determine the time interval for the integration, and how does one allow for sensitivity variations (either between instruments or as a function of time)?

Most of the entries in Table 2.2 (see also the representative plots of several distributions in Figure 3.1 refer to directly-observed peak fluxes. This is the simplest way to handle the data and one that suffers the least from the introduction of systematic errors due to unknown or imprecise corrections. Uncertainty due to background rates in the detector still remains, as with the intrinsic biases of the sampling. This will have virtually no effect on the largest events but dominate over the smallest ones. If the instrument measures spectra in detail, it is also possible to take one step away from instrumental bias by fitting a spectrum and then evaluating the spectral flux density at a well-measured photon energy, e.g., at $30 \mathrm{keV}$.

The representative distributions shown in Figure 3.1 have several common features: they match power laws well over a certain magnitude range, and they roll off towards higher and lower magnitudes. The roll-off at the low end is either due entirely to the sensitivity limit of the particular instrument, or is heavily confused by selection effects due to this limit. In particular, faint events may be missed as their emission is obscured by brighter simultaneous events. A fit to the distribution function that attempts to characterize this roll-over (e.g., a log-normal or a Weibull distribution; Parnell 2002) probably has little relevance to the physics of the events in the well-observed part of the distribution. At larger magnitudes the deficit also might have systematic errors (the saturation of a given detector would be an obvious one), but at some point a power-law fit for $\alpha<2$ will diverge unphysically. This implies the existence of an upper limit of some sort (e.g., Lingenfelter \& Hudson 1980). Alexander \& Daou (2007) analyzed $10 \mathrm{M}$ - and X-class flares, finding that each was composed of several bursts that saturated above $20 \mathrm{keV}$, suggesting a saturation of the HXR emission for electron-beams in large flares.

The sensitivity and bias effects (whether instrumental or observational) result in a clear change in the distributions at the extremes, but they can also have a more subtle effect on 

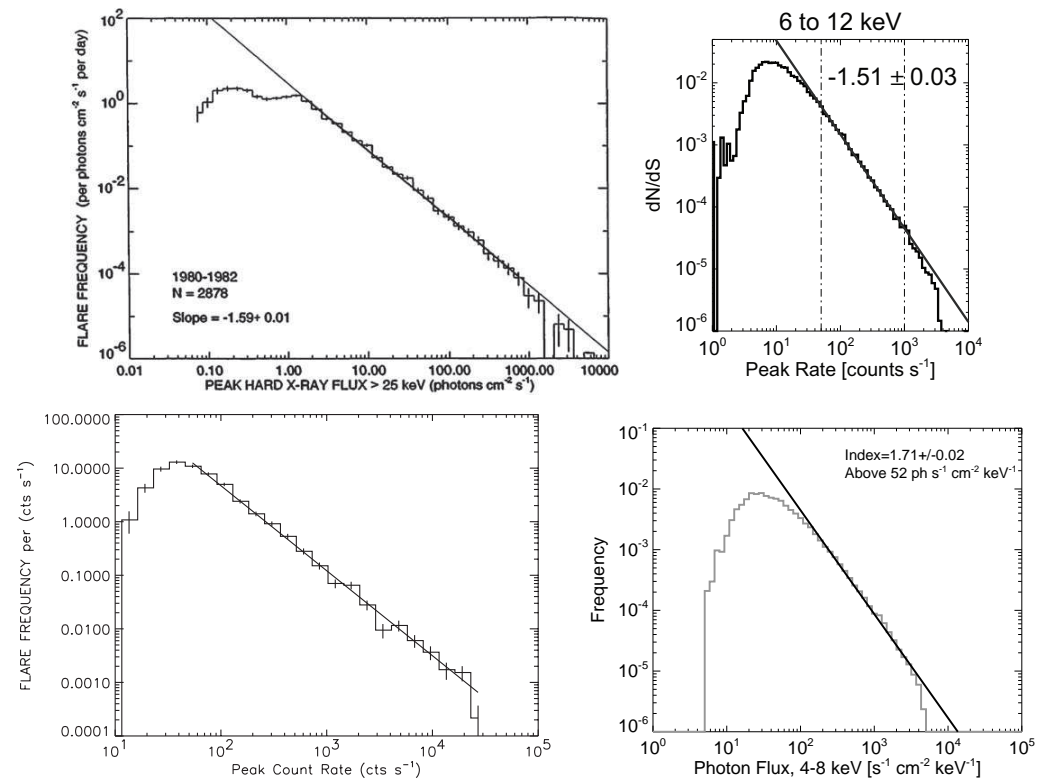

Fig. 3.1 Several of the flux distributions listed in Table 2.2 (Crosby et al. 1993; Christe et al. 2008a; Crosbv et al. 1998; Hannah et al. 2008a) (left to right, top to bottom). Another example (Veronig et al. 2002a) is shown in Figure 1.1 Reproduced by permission of the AAS.

the power law in the mid-regions. Although the power-law nature of the distribution is not dramatically changed, the value of $\alpha$ can be different (Aschwanden \& Charbonneau 2002; Aschwanden \& Parnell 2002). Some attempts have been made to correct for these biases in the context of the derived energy distributions and will be discussed further in Section 3.5

The distribution of event energies is obtained by integrating over the spectrum to estimate an energy flux, and then over time. Such conversions are not model-independent. Therefore, the direct flux measurement may be a better guide to the general conclusion from all such distributions: flare occurrence is scale-invariant. That is, the length scale does not change when multiplied by a common factor, a property of power-law distributions. Solar flares thus have behavior resembling that of earthquakes as described by the Gutenberg-Richter law (Gutenberg \& Richter 1956) but how general is it for flares? First, we note that stellar flares, on a variety of stellar types, tend to follow similar distributions (e.g. Shakhovskaia 1989). From region to region, there can be slight variations in the distribution, in particular there being a varying upper energy cutoff (Kucera et al. 1997). This is lower for smaller active regions as there is less free energy, but even in large regions there is a finite amount of energy available (making "super flares" not just infrequent but impossible). An active region from a period of low solar activity has been found to have a frequency distribution which clearly rolls over, this deviation from a power-law being attributed to the low finite energy available (Wheatland 2010).

There can also be variations on intermediate time scales (Bai 1993; Bromund et al. 1995). However, in general, these are not strong deviations from the general pattern. From RHESSI we find (Figure 3.2 right panel) little evidence for change in the slope of the microflare distribution as a function of phase of the solar cycle, with excellent statistical significance. This is consistent with the results of Veronig et al. (2002a) for GOES events 

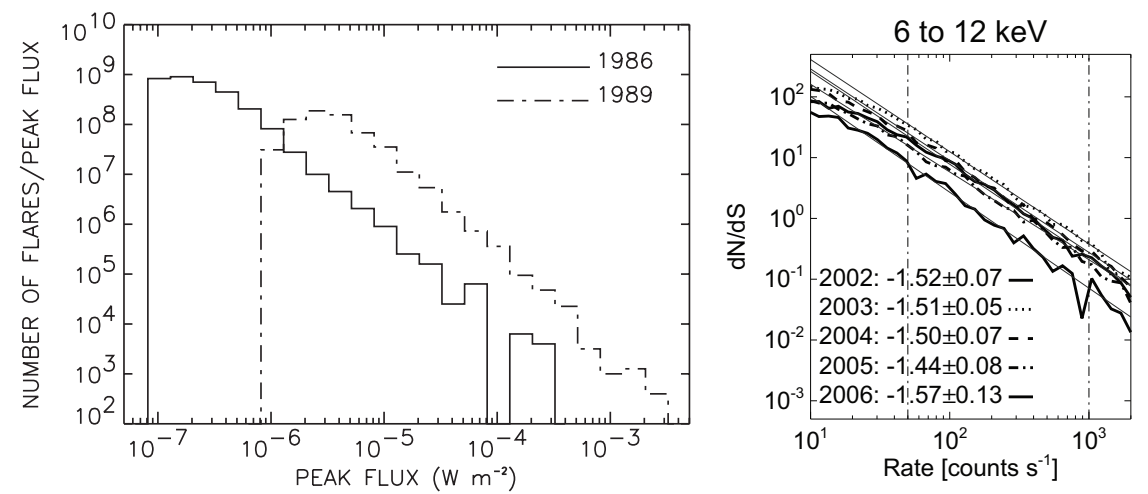

Fig. 3.2 Comparisons of occurrence distributions with phase in the solar cycle. (Left) Veronig et al. (2002a) using GOES 1-8 Å data from solar minimum (1986) to maximum (1989); (right) Christe et al. (2008a) using RHESSI microflare data from leaving solar maximum (2002) through to approaching solar minimum (2006). Reproduced by permission of the AAS.

(Figure 3.2, left panel). The scale-invariant property of the flare occurrence distributions is thought to provide the evidence that the coronal magnetic field is in a self-organized critical state (Lu \& Hamilton 1991) and this is detailed further in Section 3.3

\subsection{Time distributions}

The duration of an event must be known for an estimation of the fluence and therefore also for an estimation of the event energy. Figure 3.3 shows two examples of duration distributions: from GOES (Veronig et al. 2002a) and from RHESSI (Christe et al. 2008a). The GOES data have a clear power-law falloff to long durations (see also Drake 1971b), whereas the RHESSI distributions (shown separately for rise, fall, and total times) have more symmetrical distributions. This is because the RHESSI data are considerably more affected by selection bias. Trying to determine the duration of the flare crucially depends on being able to distinguish the flare from the background. The RHESSI distribution shown is for microflares (smaller than GOES C-class) for which it is difficult to separate their start and end times from the background. The GOES distribution is only for B-class events and larger $\left(>10^{-7} \mathrm{~W} \mathrm{~m}^{-2}\right)$ and the background rate in GOES varies less than in RHESSI.

Lee et al. (1993) explicitly deal with the simultaneous flux and duration distributions of an HXR data set, that of (ICE, formerly ISEE-3) above $26 \mathrm{keV}$ (Anderson et al. 1978). This analysis of joint variables requires an explicit consideration of the parameter-space domain as a means of understanding sampling bias. Lee et al. (1993) concluded that a correlation between the duration and peak flux was not present; this would be a requirement if microflares or nanoflares were to outweigh the energy present in the ordinary flares. Thus, the power laws observed in the distributions of peak fluxes most likely provide a guide to the "true" distribution of total event energies.

The other important temporal signature of flares is the time between one flare and the next one, the waiting-time. This is generally taken to be the time between the peak emission of one flare and the next. The waiting-time distributions provide information about the probability of a flare occurring. 

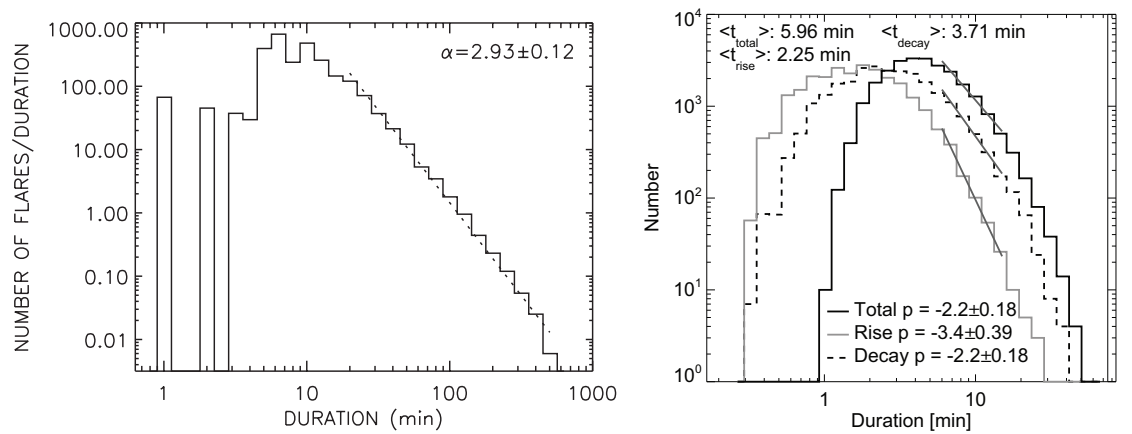

Fig. 3.3 Left: the distribution of the durations of flares GOES $>\mathrm{B}$ class Veronig et al. 2002a); right: distribution of durations of microflares (Christe et al. 2008a), typically below GOES C class. Reproduced by permission of the AAS.

These distributions can be predicted by self-organized criticality (avalanche) models of the coronal magnetic fields, discussed further in Section 3.3 These avalanche models have an exponential waiting-time distribution which corresponds to a constant Poisson flare occurrence rate (Wheatland et al. 1998). Such a flare occurrence model produces the desired power-law distribution of energy and duration. Individual active regions demonstrate waiting-time distributions that are either exponentials or the sum of exponentials Wheatland 2001; Moon et al. 2001). The latter results can be explained by the Poisson occurrence rate varying as the active region crosses the solar disk.

The waiting-time distribution found for large samples of X-ray flares is shown in Figure 3.4 The sample of 6,919 flares observed $>30 \mathrm{keV}$ with ICE/ISEE 3 is found to have a waiting-time distribution that is neither power-law nor exponential (Wheatland et al. 1998), shown left in Figure 3.4 A larger study of 32,563 GOES C-class and above flares demonstrates a power-law tail in its waiting-time distribution over long timescales Wheatland 2000), shown right in Figure 3.4 The index of this power law varies with the solar cycle (Wheatland \& Litvinenko 2002), again consistent with a Poisson occurrence with a timevarying rate. An alternative model explains this tail using an occurrence rate with a Lévy distribution (Lepreti et al. 2001) but this also requires a "memory" in the underlying process. This suggests that not only can flare rates be determined but features of the underlying physical processes can be understood, although this is still under debate. The waiting-time distribution of Type III radio bursts from an active region have been found to be consistent with a Poisson process (Eastwood et al. 2010).

An overabundance of short waiting-times compared to simulations has now been found Wheatland et al. 1998), suggesting that HXR bursts are not independent events. This sympathetic flaring behavior has frequently been suggested previously (Fritzova-Svestkova et al. 1976), referring to temporally close flares in different active regions. This may also relate to the misidentification of several peaks within a single flare as multiple events. It is difficult with non-imaging instruments to exclude closely related flares from the same active regions, but statistically significant evidence for sympathetic flaring has been found Moon et al. 2002). RHESSI would be able to provide such information, as well as investigating waitingtimes for smaller HXR flares, but the highly discontinuous nature of the data (with gaps due to nighttime, South Atlantic Anomaly passage, etc.) would make such analysis highly subject to selection effects. 

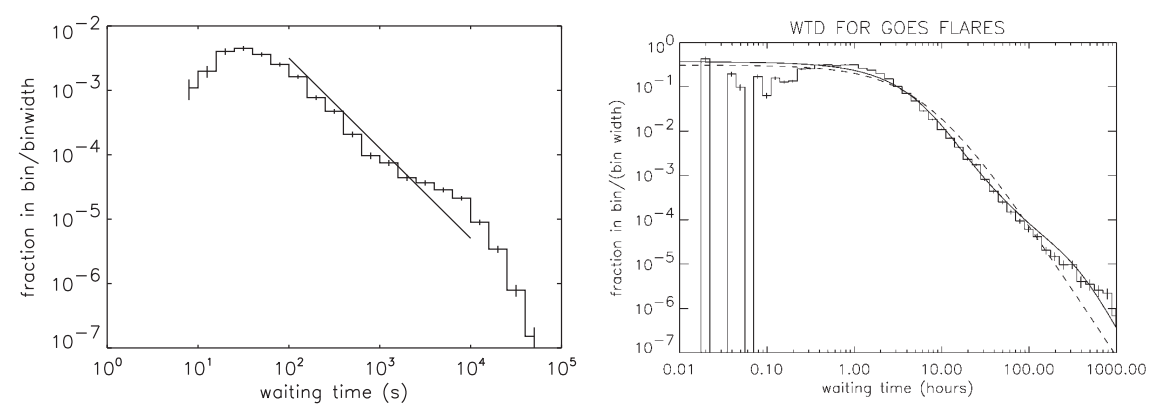

Fig. 3.4 Waiting-time distributions for different sets of X-ray flares. Left: ICE/ISEE-3 study of 6919 flares $>30 \mathrm{keV}$ (Wheatland et al. 1998), the overplotted solid line is a power-law fit. Right: 32,563 GOES flares above C-class (Wheatland 2000), the overplotted lines indicate a model for a time-dependent Poisson process (solid) and independent events with an exponential distribution of rates (dashed). Reproduced by permission of the AAS.

\subsection{Origin of power-law distribution}

An early analytic model for the statistics of solar-flare occurrence assumed that the available energy grew exponentially with time and would be released as a flare with a Poisson probability distribution (Rosner \& Vaiana 1978). This results in a power-law frequency distribution. This problem was later recast as a steady-state transport equation, allowing the inclusion of an arbitrary energy resupply rate (Litvinenko 1994). These models assume that all of the free energy is released in a flare, as in a relaxation oscillator. Such behavior has never been found in the occurrence patterns of solar flares, although there are other astrophysical contexts in which it has (e.g., Lewin et al. 1976). Wheatland \& Glukhov (1998) and Wheatland (2008) generalized these models to take into account that the flares do not release all of the free energy by finding a "master equation" to describe the system. This model again produces a power-law flare frequency distribution as well as a high-energy turnover.

A complementary view is that the scale-invariant behavior of the flare distributions implies that the system dynamics can be described as variations around a self-organized critical state (Lu \& Hamilton 1991; Lu et al. 1993). In the standard view, magnetic energy builds up in complicated and stressed magnetic field structures in the corona due to the motion and emergence of magnetic fields through the photosphere. Eventually, the coronal structure loses equilibrium catastrophically, and its restructuring (usually thought to involve magnetic reconnection) suddenly liberates some of the built-up magnetic energy. This newly released energy goes into the various forms we observe in a solar flare. In the scenario of the self-organized critical state, the instabilities are spontaneous, independent of the history of how the energy accumulated, and directly trigger a cascade of energy releases, ending in a temporarily stable state. Therefore, the same process could easily produce events at the nanoflare level as well as the more energetic flares. The avalanche-like behavior of such a system is frequently described using an idealised "cellular automaton" model (Katz 1986; Bak et al. 1987). Such a model does not depend upon the actual physical mechanisms involved at the microscopic level, replacing them with a schematic set of $a d-h o c$ rules for the system evolution. The links between the physics of such a model and its statistical description remain unclear, though taking a steady-state energy release in the "master equation" is similar to assuming an underlying avalanche process (Wheatland \& Glukhov 1998). Nev- 

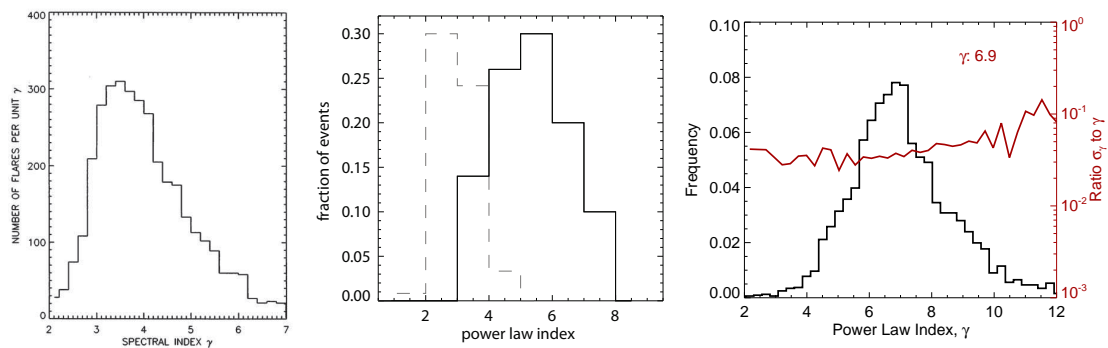

Fig. 3.5 Distributions of HXR power-law index $\gamma$ for Left: 6919 large flares observed above 30keV with ICE/ISEE-3 (Bromund et al. 1995); middle; 55 large flares on the disk (dashed grey) and occulted (solid black) with RHESSI (Krucker \& Lin 2008). Right: 9161 RHESSI microflares from the sample of Hannah et al. (2008a), the dark red line indicating the error ratio. Reproduced by permission of the AAS.
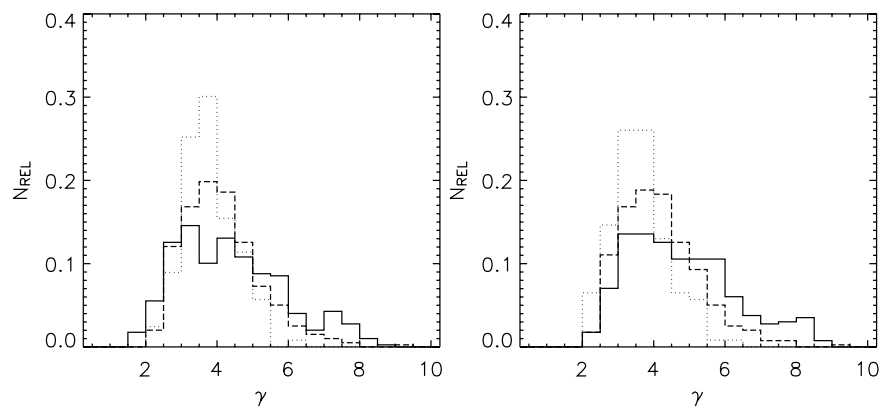

Fig. 3.6 The effect of albedo correction on the observed HXR power-law index, with histograms of $\gamma(l e f t)$ found from spectrum fitting and (right) then corrected for albedo (Kašparová et al. 2007). The different lines show the power-law fits made over different energy ranges: 398 flares with $\gamma_{0}$ (solid) 15-20 keV and $\gamma_{1}$ (dashed) 20-35 keV, and 123 flares with $\gamma_{2}$ (dotted) $35-50 \mathrm{keV}$.

ertheless an extensive literature applying cellular automata to solar flares has arisen (e.g., Charbonneau et al. 2001).

\subsection{Nonthermal and thermal spectral parameters}

The RHESSI X-ray spectra bridge the thermal and nonthermal spectral domains, at the same time providing better spectral resolution than any earlier HXR imager. The nonthermal emission of the impulsive phase is often characterized by brief spikes of emission with soft-hardsoft (steep to flat to steep) spectral evolution, and its simplest characterization is a power law in energy. The SXR thermal emission roughly follows the time integral of the HXR impulsive-phase emission, following the Neupert effect (Neupert 1968). This empirical result is thought to demonstrate that the total nonthermal energy deposited heats the chromospheric plasma, driving it up into flaring loops, producing bright thermal emission.

\subsubsection{Nonthermal}

As regards energetics, the HXR parameter of interest is the slope of the power-law fit to the photon spectrum in the impulsive phase, namely $\gamma$ in $I(\varepsilon) \propto \varepsilon^{-\gamma}$. This spectrum gives us the best possible guide to the behavior of the energy in the impulsive-phase electrons, 

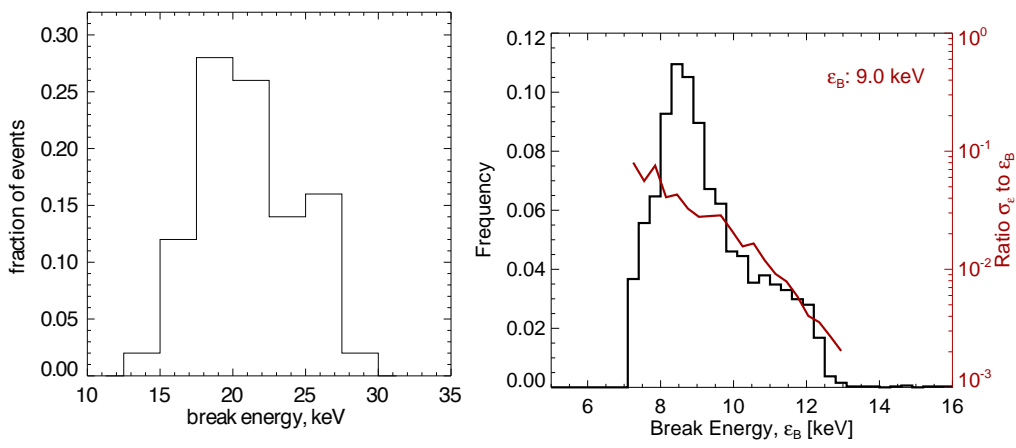

Fig. 3.7 Two examples of low-energy break energy distributions from fitting RHESSI photon flare spectra, (left) for 55 large occulted flares (Krucker \& Lin 2008) and (right) 4236 microflares (Hannah et al. 2008a), the dark red line indicating the error ratio. Reproduced by permission of the AAS.

which can be highly significant (Brown 1971; Lin \& Hudson 1976). Figure 3.5 shows the distributions of the power-law index $\gamma$ from several data sets. Each shows clearly distinguishable selection effects. The left panel of Figure 3.5 displays the spectral index of large flares above $30 \mathrm{keV}$ as found from ICE/ISEE-3 (pre-RHESSI). RHESSI observations of large flares indicate similar spectral indices, with occulted flares (those with their footpoints hidden behind the limb so the HXRs are coronal in origin) have systematically softer spectra (Krucker \& Lin 2008) as seen in the middle panel of Figure 3.5. RHESSI microflares (Figure 3.5 right panel) show many much steeper spectra which are often difficult to distinguish from the thermal emission (Hannah et al. 2008a). Kašparová et al. (2007) were able to isolate one important source of systematic error, namely the albedo resulting from X-rays backscattered off the photosphere (Santangelo et al. 1973; Kontar et al. 2010). The albedo correction affects mainly low energies, $\sim 20 \mathrm{keV}$. The result of the correction is a steeper photon spectrum at those energies, as shown in detail in Figure 3.6 RHESSI allows imaging spectroscopy of individual HXR footpoints and the difference in spectral index between footpoints in large flares (above GOES M-class) with two well-resolved footpoints ranges between 0 to 0.6 (Saint-Hilaire et al. 2008).

The essential problem in the interpretation of the hard X-ray spectrum lies in the behavior of the bremsstrahlung cross-section (e.g., Brown 1971). The photon spectrum is an integral over the electron spectrum at all higher energies. This smearing effect means that, in practice, it is difficult to determine the parent electron spectrum at low energies, and yet these lower-energy electrons contain most of the energy supplied to the flaring plasma via collisions. Analytically, the electron spectrum can be assumed to exist above some "cutoff" energy $E_{C}$, with means the accelerated electron population appears abruptly at energies where the thermal spectrum is negligible. This results in a flattening of the expected photon spectra, with a "break" energy $\varepsilon_{B}$ occurring at photon energies below the electron cutoff energy (Kosugi et al. 1988). Although the spectral indices of the source electron distributions and observed photon spectra are related in a simple way (Brown 1971; Kontar et al. 2010), this is not the case for the photon break and electron cutoff energies. There are large and ill-defined uncertainties in the inverse or forward deconvolutions of the HXR spectrum for these parameters.

Sui et al. (2007) discuss the behavior of the break energy in a selection of "early impulsive" events, which have less confusion between thermal and nonthermal components. They found that 9 out of 33 early impulsive flares demonstrated spectral flattening in the X-ray 


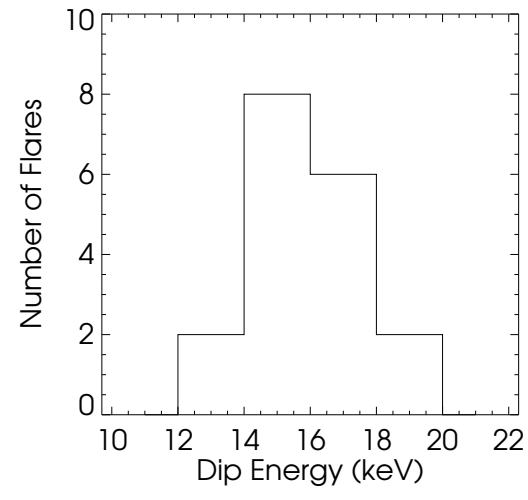

Fig. 3.8 Histogram of the measured local minimum of the dip in the mean electron flux spectrum for a sample of 18 flares (Kontar et al.2008). These dips can be removed by correcting for albedo and do not represent a low-energy cutoff.

spectrum towards low energies, a signature of a low-energy cutoff in the electron spectrum. In three of these events, albedo correction removed the flattening, but in the remaining six, the flattening was found to be consistent with forward-fitting a low-energy cutoff in the range $15-50 \mathrm{keV}$, which also correlates with the HXR flux. This is at lower energies than found from fitting the X-ray spectrum of flares without early impulsive emission.

Prior to RHESSI this confusion was not a problem, as an instrumental cutoff of around $20-30 \mathrm{keV}$ meant that the transition of the nonthermal to thermal components of the spectra was rarely observed. Figure 3.7 shows two examples of break-energy distributions derived from the RHESSI data, the left panel showing large occulted flares (Krucker \& Lin 2008) and the right panel showing microflares (Hannah et al.2008a). For microflares, the observed break tends down to very low energies where there are multiple emission lines in the thermal spectra, as can be seen in Figure 2.6. This makes it very difficult to determine $\varepsilon_{B}$ accurately. In addition, a model requiring a sharp cutoff in the nonthermal electron distribution down at low energies where the thermal spectrum is non-negligible does not seem appropriate, as a smoother transition is expected. The validity of this model and the effort to determine the energy in a microflare's nonthermal electrons is problematic and is discussed in detail in Section 3.5

Instead of trying to fit models to the photon spectrum based upon the expected analytical derivation from the electron distribution, Kontar et al. (2008) inverted the photon spectrum directly to obtain the mean electron spectrum for several flares. They found that there was no sharp cutoff in the electron distribution, but instead a dip between $12-20 \mathrm{keV}$. A histogram of these dip energies is shown in Figure 3.8 Making albedo corrections to these spectra completely removed the dip, suggesting that there is a smooth transition between the thermal and nonthermal emissions. Their study also suggests that if low-energy cutoffs exist in the mean electron spectra of these flares, they should be located at energies less than $\sim 12 \mathrm{keV}$. This implies that applying a cutoff model is only appropriate when considering an electron population well away from the thermal distribution.

\subsubsection{Thermal}

For the basic parameters of the thermal distribution, the present standard approach is to make an isothermal fit to a model spectrum derived from the Chianti atomic-physics database (Landi \& Phillips 2006), using standard assumptions about abundances and ionization states. 

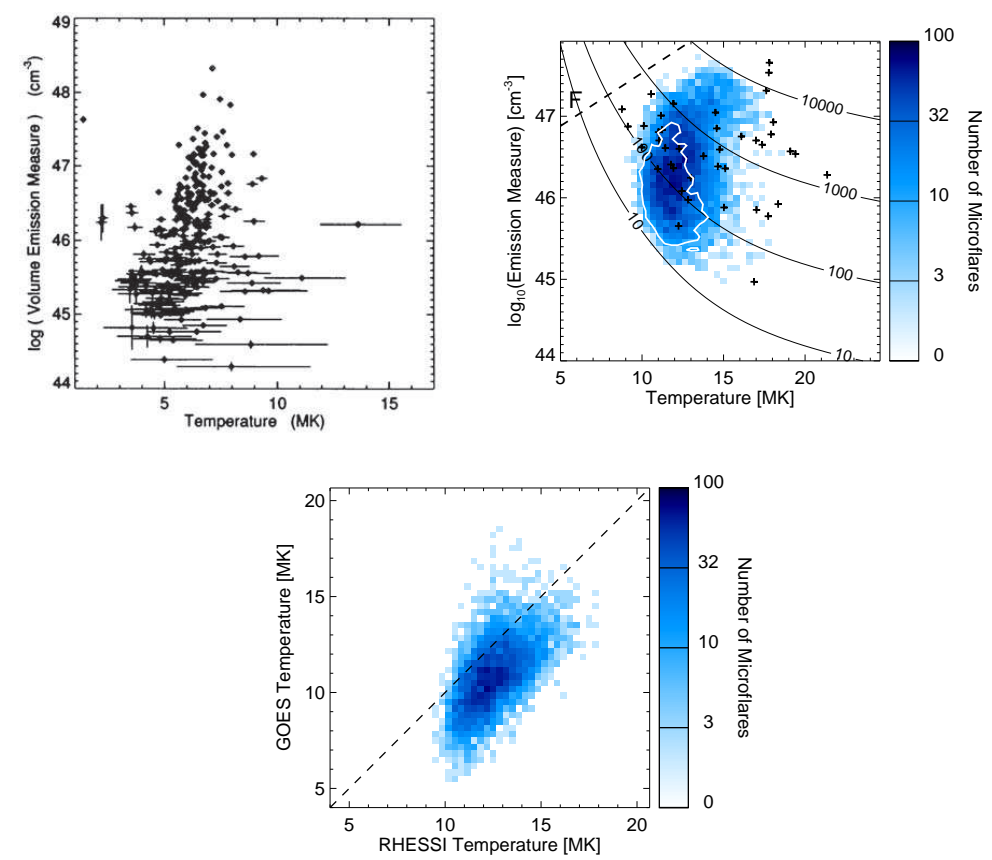

Fig. 3.9 Thermal parameter surveys of microflares. (Top left) 291 Yohkoh/SXT microflares (Shimizu 1995) (his "active region transient brightenings"). Reproduced by permission of the PASJ. (Top right) 9161 RHESSI microflares (Hannah et al. 2008a). The numbered solid lines show expected 4-8 keV RHESSI counting-rate levels as a function of model T and EM, the white contour shows the events with GOES T $<10 \mathrm{MK}$ from the bottom panel and the dashed line (F) the relationship between emission measure and temperature found by Feldman et al. (1996); see Figure 3.10 Note that neither of these studies individually confirms this correlation, and that the two data sets are almost disjoint. (Bottom) The microflare temperature derived using both RHESSI and GOES for 6740 microflares (Hannah et al. 2008a). Reproduced by permission of the AAS.

The isothermal fit determines an effective temperature $T$ and emission measure $n^{2} V$. Figure 3.9 shows comparable regions of this parameter space for microflares observed with the Yohkoh/SXT grazing-incidence telescope, at energies below about $2 \mathrm{keV}$ (Shimizu 1995), top left, and by RHESSI at energies around 6-12 keV (Hannah et al. 2008a), top right. The two samples, though taken at different times, arguably represent the same class of events, and yet the sets of points are almost disjoint. This illustrates the effects of experimental bias, in that the isothermal approximation made for each instrument will produce different weightings of the full DEM (differential emission measure) distribution. This can be further seen in the bottom panel of Figure 3.9 where the temperature has been derived for the same microflares at the same time using RHESSI and GOES separately. Clearly each instrument is responding to different parts of a DEM distribution.

Extending this to large flares, the thermal parameters continue to correlate in the sense that brighter flares have higher temperatures, but with a relatively slow growth of temperature with emission measure. Feldman et al. (1996) found it to extend over three decades of flare magnitude and a factor of five in temperature (5 to $25 \mathrm{MK}$ ) when using GOES emission measure and Yohkoh/BCS2 peak temperature estimates of $868 \mathrm{~A}$ - to X-class flares. But this

\footnotetext{
${ }^{2}$ Bent (or Bragg) Crystal Spectrometer.
} 


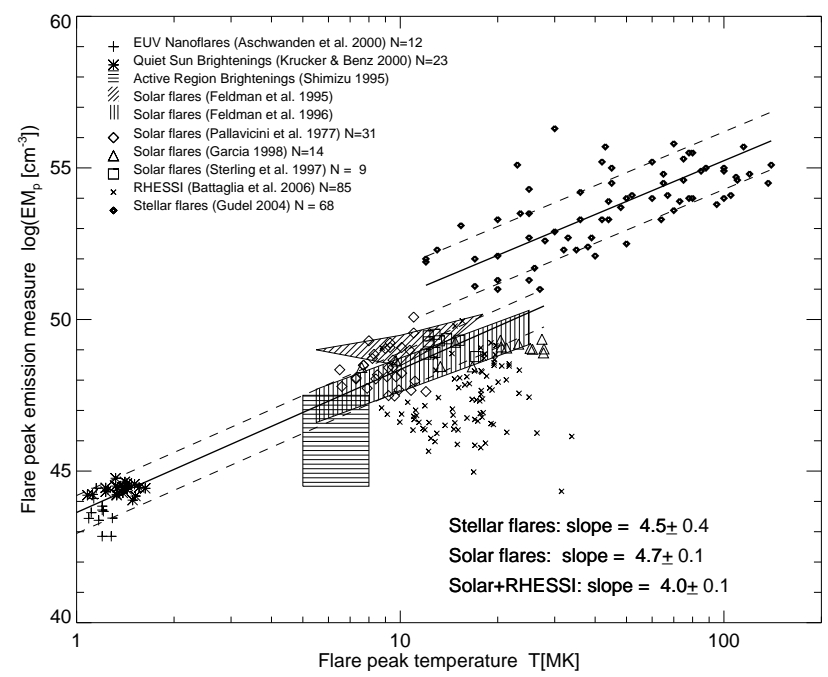

Fig. 3.10 Thermal fits from various surveys showing the correlation of emission measure (log scale) with temperature of solar flare observations compared with observations of stellar flares and with quiet-Sun brightenings, from the sources shown (Aschwanden et al. 2008). Reproduced by permission of the AAS.

correlation cleanly misses the RHESSI microflare points, as shown by the dashed line in Figure 3.9, these tend to be much hotter than predicted. The fit misses the SXT parameter space in a similar way. We presume these two samples to represent the same physical objects, but with different observational bias. Kay et al. (2003) found a similar correlation to Feldman et al. (1996) using GOES emission measure and temperature of 89 B- to X-class events. Analysis of super-hot flares (>30 MK) with RHESSI, $37 \mathrm{M}$ - and X-class flares, is able to extend parameter space up to $50 \mathrm{MK}$. The GOES emission of these events still correlates in a similar manner to temperature as in the Feldman et al. (1996) survey, but it produces a somewhat flatter correlation in linear-log space (Caspi 2010).

For individual studies over narrow ranges, there appears to be a poor correlation between temperature and emission measure, shown in Figure 3.10. A possible correlation appears if many studies over a wider range are considered (Aschwanden et al. 2008) incorporating even stellar flares and quiet-Sun brightenings. Taken together one seemingly obtains a definite correlation from sample to sample, if not within a given data set. However, this may be force-fitting a single concept to different things; a single power-law fit to all of the points would be describable approximately by $E M \propto T^{7}$, but if one ignores the quiet-Sun events one might prefer a much steeper relationship such as $E M \propto T^{15}$. Given the strong systematic biases among the different kinds of observation represented, perhaps linked only by the word "flare," it is no doubt premature to draw any strong conclusions and caution is required when making such comparisons.

\subsubsection{Thermal-nonthermal relationships}

For major flares we note that the impulsive-phase nonthermal signatures often have a simple physical relationship with the gradual thermal signatures, namely the Neupert effect where the time integral of the HXR (and microwave) emission is empirically seen to match the 

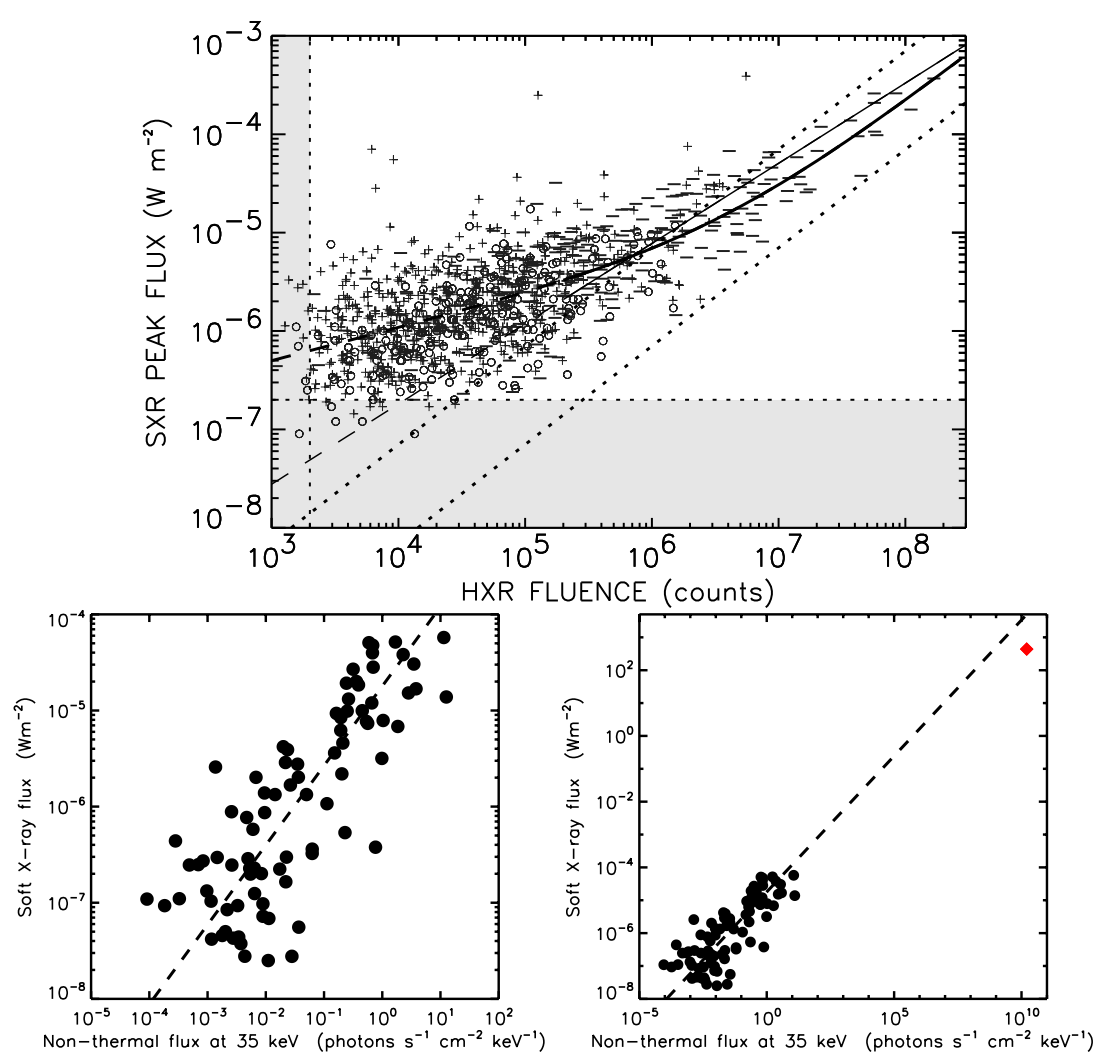

Fig. 3.11 Comparisons of thermal and nonthermal fluxes for ordinary flares. Upper: hackground-subtracted SXR peak flux from GOES vs. HXR fluence from HXRBS (Veronig et al. 2002b). The solid lines show various functional fits, the dashed lines show proportionality, with extension to smaller fluence, and the grey areas indicate regions below instrumental threshold. The different plot symbols indicate the time difference between SXR and HXR peaks. Lower: a representative sample of RHESSI flares (Battaglia et al. 2005), plus the same sample but including the enormous stellar flare observed by Osten et al. (2007) (the red point). Again, the dashed line shows proportionality.

SXR time profile (Neupert 1968; Hudson 1991a; Dennis \& Zarro 1993). Does this relationship extend into the microflare domain as well? Figure 3.11 shows how well the thermal and nonthermal signatures relate. The GOES peak flux and HXRBS (Hard X-Ray Burst Spectrometer on the Solar Maximum Mission, SMM) fluences show a large scatter for weaker events, but for the better-observed large energetic events approach a proportional relationship with little scatter (Veronig et al. 2002b). One would expect greater scatter in the fainter events purely due to selection effects, instrumental sensitivity and analysis procedure (e.g., background subtraction performed).

For RHESSI, a similar effect can be observed (Battaglia et al. 2005; Stoiser et al. 2007), as shown in the lower panel of Figure 3.11, although here the fluxes are directly compared, rather than the SXR flux and HXR fluence. Also shown is one of the most energetic stellar flares observed (Osten et al. 2007), over six orders of magnitude brighter in SXRs than the largest solar flare. Here again the SXR and HXR fluxes scaled together as in the "big flare syndrome." This relationship appears to extend into the microflare domain. Figure 3.12 shows that it does, using the large sample of RHESSI microflares studied by Hannah et al. 


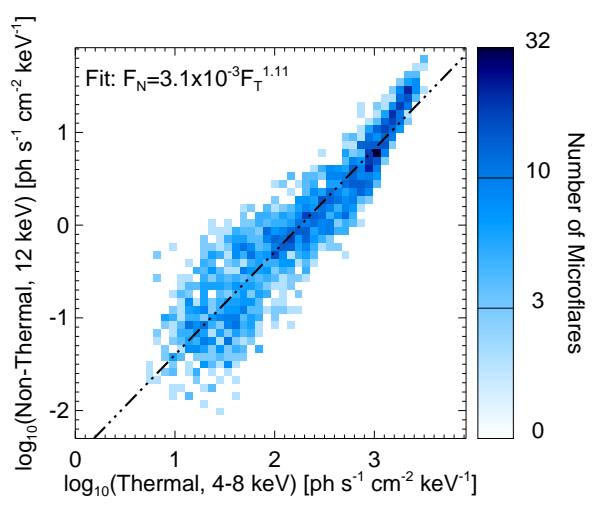

Fig. 3.12 Thermal/nothermal correlation for 4236 RHESSI microflares (Hannah et al. 2008a). The nonthermal flux is the estimated $12 \mathrm{keV}$ emission using the fitted nonthermal model. The thermal flux is found from fitting the X-ray visibilities over $4-8 \mathrm{keV}$. Note that the axes are inverted relative to those of Figure 3.11 but that the line of proportionality is the same. Reproduced by permission of the AAS.

(2008a) and Christe et al. (2008a). Note that the deviation from the trend line for the largest events is instrumental and due to increased detector deadtime before RHESSI's thin shutter deploys. In general, Figure 3.12 provides additional strong evidence that the microflares simply represent an extension, to lower energies, of the same physical processes at work in flares. Aschwanden (2007) has pointed out that such a relationship depends upon the scaling of the event environment; at some point we would expect the weakest and the most powerful events to show some kind of deviation from this behavior - for example, lowaltitude loops might have shorter coronal cooling times and therefore depart systematically from the observed thermal/nonthermal correlation.

Detailed quantitative analysis of flare thermal and nonthermal emission suggests that the underlying mechanisms are more complicated than empirically shown via the Neupert effect. The differential emission measures (DEMs) for 80 flares were studied with the Yohkoh/SXT and BCS instruments with the finding that the high-temperature plasma $(>16.5 \mathrm{MK})$ is more likely to demonstrate the Neupert effect than lower temperature plasma (McTiernan et al. 1999). Given that the Neupert effect is thought to show that the accelerated electrons are responsible for heating the chromospheric plasma, Veronig et al. 2005) investigated the similarity of the power in the electron beam compared to the power required to produce the observed SXRs. They expected the powers to be better correlated than the HXR fluence and SXR flux but found a similar correlation. One possibility for this could be that the heating of SXR-emitting plasma is not solely due to the electron beam that produces the HXR emission.

\subsection{Energy distributions}

As previously introduced, the thermal and nonthermal energies of flares cannot be determined directly from observations but have to be inferred using model assumptions. The ambiguities of these models and the errors and bias on the observational parameters result in large uncertainties in the energy, and hence in the energy distribution.

The thermal energy $U_{\text {th }}$ can be estimated via

$$
U_{\text {th }}=3 n_{\mathrm{e}} k_{\mathrm{B}} T V=3 k_{\mathrm{B}} T \sqrt{E M \cdot V_{\mathrm{obs}} f},
$$

where $V$ is the volume of the emitting plasma and $f$ is the filling factor (the ratio of the actual volume to the measured value, such that $V=f V_{\mathrm{obs}}$, where $n_{\mathrm{e}}$ is the plasma density, 
$T$ is the temperature, and $E M=n_{\mathrm{e}}^{2} V$ is the emission measure). The temperature and emission measure can be found through fitting an isothermal model spectrum to either the flare spectrum or the ratio of images in multiple wavelengths. The volume is estimated from the 2-D area of emission from the images, with some model assumption to convert to a volume. The thermal energy (given by Equation 3.1) is an upper limit estimate over the time of the observed spectrum and images, since a filling factor of $f=1$ is normally assumed (see, e.g., Fletcher et al. 2011), though in reality it could be considerably smaller, $10^{-3}$ to $10^{-2}$ (Cargill \& Klimchuk 1997; Takahashi \& Watanabe 2000).

The power in the nonthermal electrons of energies above $E_{\mathrm{C}}$ (in $\mathrm{keV}$ ) is estimated via

$$
P_{\mathrm{N}}\left(>E_{\mathrm{C}}\right)=9.5 \times 10^{24} \gamma^{2}(\gamma-1) \beta(\gamma-0.5,1.5) E_{\mathrm{C}}^{1-\gamma} I_{0} \quad \mathrm{erg} \mathrm{s}^{-1},
$$

where $\gamma$ and $I_{0}$ are the index and normalization of the power law in the observed photon spectrum (in units of photon spectral flux) and $\beta(m, n)$ is the beta function Brown 1971; Lin 1974). To estimate the energy in the nonthermal electrons $U_{\mathrm{n}}$ the total duration of the HXR emission needs to be known. The standard "cold thick-target model" model assumes a chromospheric thick target in which a beam of electrons stops, such that the thermal energy of the ambient electrons is negligible compared to the energies of the electrons emitting the HXR.

RHESSI crucially allows the thermal and nonthermal energies to be determined in a flare using a single instrument, due to the energy range it covers and to its imaging and spectroscopic capabilities. Studies of several large flares (C-, M- and X-class) have found that the thermal and nonthermal energies are the same, to within an order of magnitude (e.g., Holman et al. 2003; Saint-Hilaire \& Benz 2005). This suggests that the conversion of the energy in the accelerated particles into thermal plasma energy is highly efficient. For C- and M-class events this is typically about $10^{30} \mathrm{erg}$ (Saint-Hilaire \& Benz 2005), and at minimum about $10^{31} \mathrm{erg}$ for large X-class events (Holman et al. 2003). Another study found that the power required for the white-light emission was comparable to the electron beam power required to produce the HXR emission, but only if the cutoff was less than $25 \mathrm{keV}$ (Fletcher et al. 2007).

For microflares, the situation is more complicated, given the difficulty in determining the properties of the photon spectrum and the uncertainty this leads to in estimating the break energy. The first detailed analysis on individual RHESSI microflares (A- and B-class flares) found that the steepness of their spectra results in considerable power in the these low-energy electrons, but only estimated the energy above $25 \mathrm{keV}$ at $10^{26}-10^{27}$ erg (Krucker et al. 2002). In a larger study of microflares, where the observed break found in the photon spectrum was used as the cutoff energy, the nonthermal energy ranged over $10^{28-31} \mathrm{erg}$ (Stoiser et al. 2007). This gives an overestimate in the energy since $\varepsilon_{B}<E_{C}$, and using slightly larger values for $E_{C}$ produced typical nonthermal energies of about $10^{28} \mathrm{erg}$, similar to the thermal energies found. In the largest RHESSI microflare study (Hannah et al. 2008a) the thermal and nonthermal energies at about the time of peak emission over $6-12 \mathrm{keV}$ were found to be similar, to within an order of magnitude, once an empirical correction factor had been used to convert the measured $\varepsilon_{B}$ to $E_{C}$. This factor was found by fitting a broken power laws to model thick-target spectra with a range of indices and low energy cutoffs. Further discussion on the calculation of flare energy and the associated issues is given in Holman et al. (2010).

The distribution of these RHESSI microflare values is shown in Figure 2.1 in comparison to previous HXR nonthermal energy distributions (Crosby et al. 1993; Lin et al. 2001a), microflares from the SXR thermal distribution (Shimizu 1995) and the thermal distribution 
of EUV nanoflares. (Parnell \& Jupp 2000; Aschwanden et al. 2000; Benz \& Krucker 2002) Although such a figure nicely shows the scaling of nanoflares through microflares to large flares, and is crucial for determining coronal heating, it is dangerously deceptive. Each of these distributions was found for a different type of event, using various instruments and for different periods during the solar cycle, and so each will be affected by different types of selection effect and biases. For instance, the SXT energies (Shimizu 1995) are from 291 brightenings in one active region over five days in 1992 August, whereas the RHESSI microflare energies (Hannah et al. 2008a) are from 9161 events taken over five years, March 2002 to March 2007. This is a comparison of probably the same type of event but even then they are still observed at different wavelengths and over different time periods. Extending this comparison to EUV nanoflares is very difficult and may not even be appropriate given that we do not know if these are similar events or completely distinct physical processes. When looking at the actual values of $\alpha$ that these distributions provide, we see the striking observational result that flare peak flux distributions are power laws flatter than $\alpha=2$, a result that goes back at least to Akabane (1956). This appears to exclude nanoflares as the source of coronal heating (Hudson 1991b). On the other hand, distributions that attempt to reflect the event energies themselves have often found steeper spectra that are consistent with Parker's idea. Parnell \& Jupp (2000) found that $\alpha$ ranged from 2.1 to 2.6 for different model assumptions but was consistently in the $>2$ range. Krucker \& Benz (1998) found that $\alpha$ was between 2.3 and 2.6 but later showed that these values and those from other EUV nanoflare studies were highly dependent on the flare selection criteria (Benz \& Krucker 2002).

So, could selection effects be responsible for this discrepancy between nanoflare and larger flare distributions? Attempts have been made to recover the intrinsic distributions from the observationally derived ones (Aschwanden et al. 2000; Aschwanden \& Parnell 2002) using a technique similar to that applied to Malmquist bias, a threshold selection effect that biases galaxy number counts in cosmology (Hendry \& Simmons 1990; Willick 1994). In this situation, the counting statistics are biased because the brighter, more distant galaxies are more likely to be counted than the fainter ones. This Malmquist-bias procedure has been used on the thermal energies derived for EUV nanoflares; it takes the means and covariances of the observed data parameters and iterates them back to the intrinsic unbiased values. To do this requires model assumptions about how the parameters required to calculate the thermal energy, in Equation 3.1, relate to each other, so that the probability distribution of the intrinsic distribution can be analytically described. However, determining how these parameters scale is, in itself, subject to biases and selection effects, as discussed in Section 3.4.2 The analysis procedure may then only adjust the scalings and not test whether the parameters actually relate in such a manner. Attempting to recover the intrinsic distributions, free of instrumental and selection effect biases, should certainly be the priority of any study deriving flare distributions, but in practice this does not readily appear to be practicable given that the parameter of greatest interest, the energy, is not directly obtainable from the existing observations. An alternative approach of determining flare energy, which is much less prone to these effects, is to measure the luminous component directly via the resulting change in the total solar irradiance (TSI). This has been done for the X17 event SOL2003-10-28T11:10 using the TIM (Total Irradiance Monitor) instrument on the Solar Irradiance and Climate Experiment (SORCE) (Kopp et al. 2005), which is sensitive to the solar emission from Xrays to far infrared. The estimated total luminous energy for this flare was approximately $5 \times 10^{32} \mathrm{erg}$ (Woods et al. 2004). This technique is only appropriate at present for the largest flares, because of competition from other sources of solar variation.

Given the evidence so far, it does not appear that flare-like events can heat the quiescent corona. Moreover it seems that making the comparison of active-region and quiet-corona 
flare events is unwise given that these transient events have different populations in appear in different physical environments (Benz \& Krucker 2002).

\section{Conclusions \& discussion}

The RHESSI data have allowed us to study microflares effectively while using the same instrumentation for major events. The statistical study of solar flares has great importance in understanding the underlying processes involved in the energy release and subsequent emission we observe in the solar corona. Often, emphasis is placed on detailed multi-wavelength studies of individual events. Such studies have their merits in revealing insights to the processes in these events. However without being able to place the flare in context of other events, it is very difficult to determine whether it is typical or unusual behavior that is being studied. Only by studying events in large numbers with a minimum of selection bias can one really approach an understanding of the general physics.

The discovery that "ordinary" flare physics extends down to the tiniest events observable by RHESSI (or by GOES) allows us to conclude that such events do not explain coronal heating. Even the smallest events RHESSI observes are in the active regions and are flare-like, distinguishable as individual events. The nanoflare hypothesis instead requires an apparently continuous flare population that is many orders of magnitude smaller. They also continue to show the flat peak-flux scaling that puts most of the energy in the most powerful events, rather than the weakest ones. In a sense, this conclusion simply confirms the appearance of the GOES data - sometimes flare-dominated, and sometimes showing steady emission. This is inconsistent with the universality of the flat flare power-law distribution. Of course, numerous individually unobservable nanoflares could create the apparently steady emissions.

To make further progress in this field, three crucial things have to happen. Firstly, we need spacecraft that have a higher sensitivity, lower background and wider dynamic range than RHESSI while maintaining the energy range covered across both imaging and spectroscopy. This would allow the faintest events in active regions and the quiet Sun, as well as the fainter emission components of large flares, to be confidently analyzed. One such suitable implementation would be a HXR focusing-optics telescope dedicated to solar observations. Second, we must understand better how biased our observations are, and how we can obtain the intrinsic unbiased physics from our observations. This will require a third advance, more sophisticated modeling of these faint events to match improvements in the data and their implications.

Acknowledgements The authors thank the editor (B. R. Dennis) and the two referees for providing many constructive comments that have greatly improved this review. IGH would also like to thank the Glasgow SSH (Solar Self Help) group for their comments. This work is financially supported through NASA contract NAS 5-98033, an STFC rolling grant, and by the European Commission through the SOLAIRE Network (MTRNCT-2006-035484), and all are gratefully acknowledged. JK acknowledges support from Grant 205/06/P135 of the GA CR and the research plan AV0Z10030501.

\section{A Determining distribution parameters}

For a power-law distribution (Equation 1.1, the standard way to determine the index $\alpha$ is to perform a linear fit to the log-log histogram of the data. However this is a highly subjective approach as there is considerable choice as to the "best" bin width and fitting method. An alternative and more objective approach is to estimate the power-law index using the maximum-likelihood method (Crawford et al. 1970; Bai 1993). This approach leads to a remarkably simple calculation on the sample to determine the index above some chosen threshold: 


$$
\alpha_{\mathrm{m}}=\frac{N}{\sum_{i=0}^{N} \ln \left(U_{i} / U_{0}\right)}+1
$$

In this example, the energies $U$ are used, where $N$ is the total number of events and $U_{i}$ is the energy of the $i^{\text {th }}$ event normalized by a threshold energy of $U_{0}$. The error in this most likely value of $\alpha_{\mathrm{m}}$ can be estimated Wheatland 2004) as

$$
\sigma_{\alpha}=\left(\alpha_{\mathrm{m}}-1\right) N^{-1 / 2} .
$$

The observed distribution, however, is often affected by instrumental and selection effects, resulting in a deviation from a power law, so fitting a power law alone would be unwise. A common problem is that the smallest events are hard to detect and analyze successfully, resulting in a flattening of the power-law for these missing smallest events. To fit this observed biased distribution a skew-Laplace distribution can be used instead of a single power law (Parnell \& Jupp 2000). This fits the desired power-law distribution for the larger events, but below some critical value, a power law with a different index is fitted to the under-reported smallest events. The determination of the parameters of this skew-Laplace distribution again can be found using a maximum likelihood estimation method (Parnell \& Jupp 2000).

Another possible distribution to describe the observed sample of data is the Weibull distribution (Parnell 2002). This distribution has the form

$$
f(x ; \kappa, \xi)=C(x / \xi)^{(\kappa-1)} \exp \left[-(x / \xi)^{\kappa}\right],
$$

where $\kappa$ is the shape parameter and $\xi$ is the scale parameter. For a shape parameter $\kappa<1$, the resulting distribution is similar to a power law, but it turns over at the smallest and largest events. This can then represent deficiencies in the smallest and largest events. Again, the under-reporting of the smallest events is likely to arise from instrumental sensitivity and selection effect bias. The largest events may be missing due to the limited dynamic range of the instrument, if these events saturate the detector; or, there might actually be fewer of these events if a critical physical upper limit is being reached (for instance maximum energy available in an active region (Kucera et al. 1997). Such a feature is consistent with the predictions of avalanche models (Lu et al. 1993), as discussed in Section 3.3 The parameters of this Weibull distribution can be determined again using the maximum likelihood method (Parnell 2002).

As there are several distributions that could successfully fit the data, a statistical test is needed to determine which is best. Such a test is the Kolmogorov-Smirnov statistic (KS), which is the maximum difference between the cumulative distribution function (CDF) and the empirical distribution function (EDF) (Press et al. 1992). For a data set of, energies $U$, the CDF for the $i^{\text {th }}$ energy $U_{i}$ is the integral of the PDF, using the fitted parameters, to $U_{i}$. The EDF is derived from the observed/calculated parameters and in this example is the number of events with energy less than or equal to $U_{i}$, which turns out to be $(i-1 / 2) / N$. The KS statistic then provides a measure of the significance level of each distribution (Press et al. 1992). Plotting CDF versus EDF provides a graphical way of determining how consistently the data belong to the chosen distribution. A graph similar to the familiar histogram can be obtained by plotting 1- CDF and EDF against the event parameters (i.e., energy).

A detailed example of using the maximum likelihood method to determine the parameters, and testing the goodness of the fit using the KS statistic for power-law and Weibull distributions in the solar context, is given in Parnell (2002).

\section{References}

W. P. Abbett, S. L. Hawley, Astrophys. J. 521, 906 (1999), doi:10.1086/307576 I. A. Ahmad, D. F. Webb, Solar Phys. 58, 323 (1978), doi:10.1007/BF00157278

K. Akabane, Pub. Astron. Soc. Japan 8, 173 (1956)

D. Alexander, A. G. Daou, Astrophys. J. 666, 1268 (2007), doi:10.1086/520331

K. A. Anderson, S. R. Kane, J. H. Primbsch, R. H. Weitzmann, W. D. Evans, R. W. Klebesadel, W. P. Aiello, IEEE Transactions on Geoscience Electronics 16, 157 (1978)

M. J. Aschwanden, Advances in Space Research 39, 1867 (2007), doi:10.1016/j.asr.2007.03.050

M. J. Aschwanden, P. Charbonneau, Astrophys. J. Lett. 566, L59 (2002), doi:10.1086/339451

M. J. Aschwanden, B. R. Dennis, A. O. Benz, Astrophys. J. 497, 972 (1998), doi:10.1086/305484

M. J. Aschwanden, C. E. Parnell, Astrophys. J. 572, 1048 (2002), doi:10.1086/340385

M. J. Aschwanden, R. A. Stern, M. Güdel, Astrophys. J. 672, 659 (2008), arXiv:0710.2563 doi:10.1086/523926 
M. J. Aschwanden, T. D. Tarbell, R. W. Nightingale, C. J. Schrijver, A. Title, C. C. Kankelborg, P. Martens, H. P. Warren, Astrophys. J. 535, 1047 (2000)

H. Aurass, K.-L. Klein, P. C. H. Martens, Solar Phys. 155, 203 (1994), doi:10.1007/BF00670741

T. Bai, Astrophys. J. 404, 805 (1993), doi:10.1086/172335

P. Bak, C. Tang, K. Wiesenfeld, Physical Review Letters 59, 381 (1987), doi:10.1103/PhysRevLett.59.381

M. Battaglia, P. C. Grigis, A. O. Benz, Astron. Astrophys. 439, 737 (2005), arXiv:astro-ph/0505154 doi:10.1051/0004-6361:20053027

A. O. Benz, S. Krucker, Astrophys. J. 568, 413 (2002)

D. A. Biesecker, On the Occurrence of Solar Flares Observed with the Burst and Transient Source Experiment, Ph.D. thesis, AA(UNIVERSITY OF NEW HAMPSHIRE.) (1994)

P. L. Bornmann, Astrophys. J. 356, 733 (1990), doi:10.1086/168880

K. R. Bromund, J. M. McTiernan, S. R. Kane, Astrophys. J. 455, 733 (1995), doi:10.1086/176619

J. W. Brosius, K. J. H. Phillips, Astrophys. J. 613, 580 (2004), doi:10.1086/422873

J. C. Brown, Solar Phys. 18, 489 (1971)

P. J. Cargill, J. A. Klimchuk, Astrophys. J. 478, 799 (1997), doi:10.1086/303816

P. J. Cargill, J. A. Klimchuk, Astrophys. J. 605, 911 (2004), doi:10.1086/382526

R. C. Carrington, Mon. Not. Roy. Astron. Soc. 20, 13 (1859)

A. Caspi, Super-hot $(T>30$ MK) Thermal Plasma in Solar Flares, Ph.D. thesis, University of California, Berkeley (2010)

P. Charbonneau, S. W. McIntosh, H.-L. Liu, T. J. Bogdan, Solar Phys. 203, 321 (2001), doi:10.1023/A:1013301521745

C. Chifor, H. Isobe, H. E. Mason, I. G. Hannah, P. R. Young, G. Del Zanna, S. Krucker, K. Ichimoto, Y. Katsukawa, T. Yokoyama, Astron. Astrophys. 491, 279 (2008), doi:10.1051/0004-6361:200810265

S. Christe, I. G. Hannah, S. Krucker, J. McTiernan, R. P. Lin, Astrophys. J. 677, 1385 (2008a), doi:10.1086/529011

S. Christe, S. Krucker, R. P. Lin, Astrophys. J. Lett. 680, L149 (2008b), doi:10.1086/589971

J. W. Cirtain, L. Golub, L. Lundquist, A. van Ballegooijen, A. Savcheva, M. Shimojo, E. DeLuca, S. Tsuneta, T. Sakao, K. Reeves, M. Weber, R. Kano, N. Narukage, K. Shibasaki, Science 318, 1580 (2007), doi:10.1126/science. 1147050

E. W. Cliver, L. Svalgaard, Solar Phys. 224, 407 (2004), doi:10.1007/s11207-005-4980-z

D. F. Crawford, D. L. Jauncey, H. S. Murdoch, Astrophys. J. 162, 405 (1970), doi:10.1086/150672

N. Crosby, N. Vilmer, N. Lund, R. Sunyaev, Astron. Astrophys. 334, 299 (1998)

N. B. Crosby, M. J. Aschwanden, B. R. Dennis, Solar Phys. 143, 275 (1993)

D. W. Datlowe, M. J. Elcan, H. S. Hudson, Solar Phys. 39, 155 (1974)

B. R. Dennis, Solar Phys. 100, 465 (1985)

B. R. Dennis, D. M. Zarro, Solar Phys. 146, 177 (1993)

J. F. Drake, Solar Phys. 16, 152 (1971a), doi:10.1007/BF00154510

J. F. Drake, Solar Phys. 16, 152 (1971b)

J. P. Eastwood, M. S. Wheatland, H. S. Hudson, S. Krucker, S. D. Bale, M. Maksimovic, K. Goetz, J. Bougeret, Astrophys. J. Lett. 708, L95 (2010), 0911.4131. doi:10.1088/2041-8205/708/2/L95

A. G. Emslie, B. R. Dennis, G. D. Holman, H. S. Hudson, Journal of Geophysical Research (Space Physics) 110(A9), 11103 (2005), doi:10.1029/2005JA011305

U. Feldman, G. A. Doschek, W. E. Behring, K. J. H. Phillips, Astrophys. J. 460, 1034 (1996), doi:10.1086/177030

U. Feldman, G. A. Doschek, J. A. Klimchuk, Astrophys. J. 474, 511 (1997), doi:10.1086/303428

G. H. Fisher, R. C. Canfield, A. N. McClymont, Astrophys. J. 289, 434 (1985), doi:10.1086/162903

L. Fletcher, B. R. Dennis, H. S. Hudson, S. Krucker, K. Phillips, A. Veronig, M. Battaglia, L. Bone, Q. Chen, P. Gallagher, P. Grigis, H. Ji, H. Liu, R. O. Milligan, M. Temmer, Space Science Reviews (2011), arXiv:XXX

L. Fletcher, I. G. Hannah, H. S. Hudson, T. R. Metcalf, Astrophys. J. 656, 1187 (2007), doi:10.1086/510446

L. Fritzova-Svestkova, R. C. Chase, Z. Svestka, Solar Phys. 48, 275 (1976), doi:10.1007/BF00151996

E. Fuerst, A. O. Benz, W. Hirth, Astron. Astrophys. 107, 178 (1982)

D. E. Gary, M. D. Hartl, T. Shimizu, Astrophys. J. 477, 958 (1997), doi:10.1086/303748

L. Golub, A. S. Krieger, J. K. Silk, A. F. Timothy, G. S. Vaiana, Astrophys. J. Lett. 189, L93+ (1974)

N. Gopalswamy, T. E. W. Payne, E. J. Schmahl, M. R. Kundu, J. R. Lemen, K. T. Strong, R. C. Canfield, J. de La Beaujardiere, Astrophys. J. 437, 522 (1994), doi:10.1086/175015

J. A. Grayson, S. Krucker, R. P. Lin, Astrophys. J. 707, 1588 (2009), doi:10.1088/0004-637X/707/2/1588

B. Gutenberg, C. F. Richter, Seismicity of the Earth and Associated Phenomena, 2nd ed. (Princeton, Princeton University Press, 1956) 
I. G. Hannah, S. Christe, S. Krucker, G. J. Hurford, H. S. Hudson, R. P. Lin, Astrophys. J. 677, 704 (2008a), arXiv:0712.2544 doi:10.1086/529012

I. G. Hannah, H. S. Hudson, G. J. Hurford, R. P. Lin, Astrophys. J. (2010)

I. G. Hannah, G. J. Hurford, H. S. Hudson, R. P. Lin, Review of Scientific Instruments 78, 4501 (2007a), doi:10.1063/1.2437120

I. G. Hannah, G. J. Hurford, H. S. Hudson, R. P. Lin, K. van Bibber, Astrophys. J. Lett. 659, L77 (2007b), arXiv: astro-ph/0702726 doi:10.1086/516750

I. G. Hannah, S. Krucker, H. S. Hudson, S. Christe, R. P. Lin, Astron. Astrophys. 481, L45 (2008b), 0712.0369 doi:10.1051/0004-6361:20079019

H. Hara, K. Nakakubo-Morimoto, Astrophys. J. 589, 1062 (2003), doi:10.1086/374778

M. A. Hendry, J. F. L. Simmons, Astron. Astrophys. 237, 275 (1990)

R. Hodgson, Mon. Not. Roy. Astron. Soc. 20, 15 (1859)

G. D. Holman, M. J. Aschwanden, H. Aurass, M. Battaglia, P. C. Grigis, E. P. Kontar, W. Liu, P. Saint-Hilaire, V. V. Zharkov, Space Science Reviews (2010), arXiv:XXX

G. D. Holman, L. Sui, R. A. Schwartz, A. G. Emslie, Astrophys. J. Lett. 595, L97 (2003), doi:10.1086/378488

H. S. Hudson, in Bulletin of the American Astronomical Society (1991a), volume 23 of Bulletin of the American Astronomical Society, pp. 1064-+

H. S. Hudson, Solar Phys. 133, 357 (1991b)

H. S. Hudson, Astrophys. J. Lett. 663, L45 (2007), 0707.1118 doi:10.1086/519923

H. S. Hudson, L. E. Peterson, D. A. Schwartz, Astrophys. J. 157, 389 (1969)

S. W. Kahler, J. Geophys. Res. 87, 3439 (1982), doi:10.1029/JA087iA05p03439

J. I. Katz, J. Geophys. Res. 91, 10412 (1986), doi:10.1029/JB091iB10p10412

J. Kašparová, E. P. Kontar, J. C. Brown, Astron. Astrophys. 466, 705 (2007), arXiv: astro-ph/0701871 doi:10.1051/0004-6361:20066689

H. R. M. Kay, J. L. Culhane, L. K. Harra, S. A. Matthews, Advances in Space Research 32, 1051 (2003), doi:10.1016/S0273-1177(03)00308-9

A. L. Kiplinger, Astrophys. J. 453, 973 (1995), doi:10.1086/176457

A. L. Kiplinger, H. A. Garcia, in Bulletin of the American Astronomical Society (2004), volume 36 of Bulletin of the American Astronomical Society, pp. 739-+

E. Kontar, J. Brown, A. Emslie, W. Hajdas, G. Holman, G. Hurford, J. Kasparova, P. Mallik, A. Massone, M. McConnell, M. Piana, M. Prato, E. Schmahl, E. Suarez-Garcia, Space Science Reviews (2010), arXiv:XXX

E. P. Kontar, E. Dickson, J. Kašparová, Solar Phys. 252, 139 (2008), 0805.1470 doi:10.1007/s11207-0089249-X

G. Kopp, G. Lawrence, G. Rottman, Solar Phys. 230, 129 (2005), doi:10.1007/s11207-005-7433-9

T. Kosugi, B. R. Dennis, K. Kai, Astrophys. J. 324, 1118 (1988), doi:10.1086/165967

J. Kotoku, R. Kano, S. Tsuneta, Y. Katsukawa, T. Shimizu, T. Sakao, K. Shibasaki, E. E. Deluca, K. E. Korreck, L. Golub, A. Bobra, Pub. Astron. Soc. Japan 59, 735 (2007)

S. Krucker, A. O. Benz, Astrophys. J. Lett. 501, L213+ (1998), doi:10.1086/311474

S. Krucker, A. O. Benz, T. S. Bastian, L. W. Acton, Astrophys. J. 488, 499 (1997), doi:10.1086/304686

S. Krucker, S. Christe, R. P. Lin, G. J. Hurford, R. A. Schwartz, Solar Phys. 210, 445 (2002), doi:10.1023/A:1022404512780

S. Krucker, R. P. Lin, Astrophys. J. 673, 1181 (2008), doi:10.1086/524010

T. A. Kucera, B. R. Dennis, R. A. Schwartz, D. Shaw, Astrophys. J. 475, 338 (1997), doi:10.1086/303532

M. R. Kundu, J. P. Raulin, N. Nitta, H. S. Hudson, M. Shimojo, K. Shibata, A. Raoult, Astrophys. J. Lett. 447, L135+ (1995), doi:10.1086/309567

M. R. Kundu, E. J. Schmahl, P. C. Grigis, V. I. Garaimov, K. Shibasaki, Astron. Astrophys. 451, 691 (2006), doi:10.1051/0004-6361:20053987

M. R. Kundu, G. Trottet, V. I. Garaimov, P. C. Grigis, E. J. Schmahl, Advances in Space Research 35, 1778 (2005), doi:10.1016/j.asr.2005.04.052

E. Landi, K. J. H. Phillips, Astrophys. Suppl. 166, 421 (2006), doi:10.1086/506180

T. T. Lee, V. Petrosian, J. M. McTiernan, Astrophys. J. 412, 401 (1993), doi:10.1086/172929

T. T. Lee, V. Petrosian, J. M. McTiernan, Astrophys. J. 448, 915 (1995), doi:10.1086/176020

F. Lepreti, V. Carbone, P. Veltri, Astrophys. J. Lett. 555, L133 (2001), doi:10.1086/323178

W. H. G. Lewin, J. Doty, G. W. Clark, S. A. Rappaport, H. V. D. Bradt, R. Doxsey, D. R. Hearn, J. A. Hoffman, J. G. Jernigan, F. K. Li, W. Mayer, J. McClintock, F. Primini, J. Richardson, Astrophys. J. Lett. 207, L95 (1976), doi:10.1086/182188

R. P. Lin, Space Science Reviews 16, 189 (1974), doi:10.1007/BF00240886

R. P. Lin, B. R. Dennis, G. J. Hurford, D. M. Smith, A. Zehnder, P. R. Harvey, D. W. Curtis, D. Pankow, P. Turin, M. Bester, A. Csillaghy, M. Lewis, N. Madden, H. F. van Beek, M. Appleby, T. Raudorf, J. Mc- 
Tiernan, R. Ramaty, E. Schmahl, R. Schwartz, S. Krucker, R. Abiad, T. Quinn, P. Berg, M. Hashii, R. Sterling, R. Jackson, R. Pratt, R. D. Campbell, D. Malone, D. Landis, C. P. Barrington-Leigh, S. Slassi-Sennou, C. Cork, D. Clark, D. Amato, L. Orwig, R. Boyle, I. S. Banks, K. Shirey, A. K. Tolbert, D. Zarro, F. Snow, K. Thomsen, R. Henneck, A. McHedlishvili, P. Ming, M. Fivian, J. Jordan, R. Wanner, J. Crubb, J. Preble, M. Matranga, A. Benz, H. Hudson, R. C. Canfield, G. D. Holman, C. Crannell, T. Kosugi, A. G. Emslie, N. Vilmer, J. C. Brown, C. Johns-Krull, M. Aschwanden, T. Metcalf, A. Conway, Solar Phys. 210, 3 (2002), doi:10.1023/A:1022428818870

R. P. Lin, P. T. Feffer, R. A. Schwartz, Astrophys. J. Lett. 557, L125 (2001a)

R. P. Lin, P. T. Feffer, R. A. Schwartz, Astrophys. J. Lett. 557, L125 (2001b), doi:10.1086/323270

R. P. Lin, H. S. Hudson, Solar Phys. 50, 153 (1976), doi:10.1007/BF00206199

R. P. Lin, R. A. Schwartz, S. R. Kane, R. M. Pelling, K. C. Hurley, Astrophys. J. 283, 421 (1984), doi:10.1086/162321

R. E. Lingenfelter, H. S. Hudson, in The Ancient Sun: Fossil Record in the Earth, Moon and Meteorites, ed. by R. O. Pepin, J. A. Eddy, R. B. Merrill (1980), pp. 69-79

Y. E. Litvinenko, Solar Phys. 151, 195 (1994), doi:10.1007/BF00654092

C. Liu, J. Qiu, D. E. Gary, S. Krucker, H. Wang, Astrophys. J. 604, 442 (2004), doi:10.1086/381799

E. T. Lu, R. J. Hamilton, Astrophys. J. Lett. 380, L89 (1991), doi:10.1086/186180

E. T. Lu, R. J. Hamilton, J. M. McTiernan, K. R. Bromund, Astrophys. J. 412, 841 (1993), doi: $10.1086 / 172966$

K. G. McCracken, D. F. Smart, M. A. Shea, G. A. M. Dreschhoff, in International Cosmic Ray Conference (2001), volume 8 of International Cosmic Ray Conference, pp. 3209-+

J. M. McTiernan, Astrophys. J. 697, 94 (2009), doi:10.1088/0004-637X/697/1/94

J. M. McTiernan, G. H. Fisher, P. Li, Astrophys. J. 514, 472 (1999), doi:10.1086/306924

R. O. Milligan, private communication (2009)

Y.-J. Moon, G. S. Choe, Y. D. Park, H. Wang, P. T. Gallagher, J. Chae, H. S. Yun, P. R. Goode, Astrophys. J. 574, 434 (2002), doi:10.1086/340945

Y.-J. Moon, G. S. Choe, H. S. Yun, Y. D. Park, J. Geophys. Res. 106, 29951 (2001), doi:10.1029/2000JA000224

D. Moses, J. W. Cook, J.-D. F. Bartoe, G. E. Brueckner, K. P. Dere, D. F. Webb, J. M. Davis, J. W. Harvey, F. Recely, S. F. Martin, H. Zirin, Astrophys. J. 430, 913 (1994), doi:10.1086/174461

W. M. Neupert, Astrophys. J. Lett. 153, L59+ (1968)

H. W. Newton, Mon. Not. Roy. Astron. Soc. 103, 244 (1943)

A. Nindos, M. R. Kundu, S. M. White, Astrophys. J. 513, 983 (1999), doi:10.1086/306886

N. Nitta, Astrophys. J. 491, 402 (1997), doi:10.1086/304938

N. Nitta, T. S. Bastian, M. J. Aschwanden, K. L. Harvey, K. T. Strong, Pub. Astron. Soc. Japan 44, L167 (1992)

N. Nitta, T. Kosugi, Solar Phys. 105, 73 (1986), doi:10.1007/BF00156378

R. A. Osten, S. Drake, J. Tueller, J. Cummings, M. Perri, A. Moretti, S. Covino, Astrophys. J. 654, 1052 (2007), arXiv:astro-ph/0609205 doi:10.1086/509252

E. N. Parker, Astrophys. J. 330, 474 (1988), doi:10.1086/166485

C. E. Parnell, Mon. Not. Roy. Astron. Soc. 335, 389 (2002), doi:10.1046/j.1365-8711.2002.05618.x

C. E. Parnell, P. E. Jupp, Astrophys. J. 529, 554 (2000), doi:10.1086/308271

K. J. H. Phillips, Astrophys. J. 605, 921 (2004), doi:10.1086/382523

M. Pick, N. Vilmer, Astron. Astrophys. Rev. pp. 6-+ (2008), doi:10.1007/s00159-008-0013-x

J. G. Porter, R. L. Moore, E. J. Reichmann, O. Engvold, K. L. Harvey, Astrophys. J. 323, 380 (1987), doi:10.1086/165835

W. H. Press, S. A. Teukolsky, W. T. Vetterling, B. P. Flannery, Numerical recipes in C. The art of scientific computing (Cambridge: University Press, —c1992, 2nd ed., 1992)

J. Qiu, C. Liu, D. E. Gary, G. M. Nita, H. Wang, Astrophys. J. 612, 530 (2004), doi:10.1086/422401

J.-P. Raulin, S. M. White, M. R. Kundu, A. V. R. Silva, K. Shibasaki, Astrophys. J. 522, 547 (1999), doi: $10.1086 / 322974$

D. V. Reames, Space Science Reviews 90, 413 (1999), doi:10.1023/A:1005105831781

R. S. Richardson, Pub. Astron. Soc. Pac. 56, 156 (1944)

R. Rosner, G. S. Vaiana, Astrophys. J. 222, 1104 (1978), doi:10.1086/156227

P. Saint-Hilaire, A. O. Benz, Astron. Astrophys. 435, 743 (2005), doi:10.1051/0004-6361:20041918

P. Saint-Hilaire, S. Krucker, R. P. Lin, Solar Phys. 250, 53 (2008), doi:10.1007/s11207-008-9193-9

R. Saldanha, S. Krucker, R. P. Lin, Astrophys. J. 673, 1169 (2008), doi:10.1086/524929

N. Santangelo, H. Horstman, E. Horstman-Moretti, Solar Phys. 29, 143 (1973), doi:10.1007/BF00153445

A. Savcheva, J. Cirtain, E. E. Deluca, L. L. Lundquist, L. Golub, M. Weber, M. Shimojo, K. Shibasaki, T. Sakao, N. Narukage, S. Tsuneta, R. Kano, Pub. Astron. Soc. Japan 59, 771 (2007) 
A. Schadee, C. de Jager, Z. Svestka, Solar Phys. 89, 287 (1983), doi:10.1007/BF00217252

R. A. Schwartz, B. R. Dennis, G. J. Fishman, C. A. Meegan, R. B. Wilson, W. S. Paciesas, in The Compton Observatory Science Workshop, ed. by C. R. Shrader, N. Gehrels, B. Dennis (1992), pp. 457-468

N. I. Shakhovskaia, Solar Phys. 121, 375 (1989), doi:10.1007/BF00161707

K. Shibata, Y. Ishido, L. W. Acton, K. T. Strong, T. Hirayama, Y. Uchida, A. H. McAllister, R. Matsumoto, S. Tsuneta, T. Shimizu, H. Hara, T. Sakurai, K. Ichimoto, Y. Nishino, Y. Ogawara, Pub. Astron. Soc. Japan 44, L173 (1992)

T. Shimizu, Pub. Astron. Soc. Japan 47, 251 (1995)

T. Shimizu, R. A. Shine, A. M. Title, T. D. Tarbell, Z. Frank, Astrophys. J. 574, 1074 (2002), doi:10.1086/340998

M. Shimojo, S. Hashimoto, K. Shibata, T. Hirayama, H. S. Hudson, L. W. Acton, Pub. Astron. Soc. Japan 48, 123 (1996)

M. Shimojo, N. Narukage, R. Kano, T. Sakao, S. Tsuneta, K. Shibasaki, J. W. Cirtain, L. L. Lundquist, K. Reeves, A. Savcheva, Pub. Astron. Soc. Japan 59, 745 (2007)

A. V. R. Silva, H. Wang, D. E. Gary, Astrophys. J. 545, 1116 (2000), doi:10.1086/317822

H. J. Smith, E. V. P. Smith, Solar flares (New York, Macmillan, 1963)

S. Stoiser, A. M. Veronig, H. Aurass, A. Hanslmeier, Solar Phys. 246, 339 (2007), doi:10.1007/s11207-0079066-7

K. T. Strong, K. Harvey, T. Hirayama, N. Nitta, T. Shimizu, S. Tsuneta, Pub. Astron. Soc. Japan 44, L161 (1992)

L. Sui, G. D. Holman, B. R. Dennis, Astrophys. J. 670, 862 (2007), doi:10.1086/522198

M. Takahashi, T. Watanabe, Advances in Space Research 25, 1833 (2000), doi:10.1016/S02731177(99)00628-6

R. J. Thomas, R. G. Teske, Solar Phys. 16, 431 (1971), doi:10.1007/BF00162486

S. Tsuneta, J. R. Lemen, in Physics of Solar and Stellar Coronae: G. S. Vaiana Memorial Symposium, Palermo, Italy, ed. by J. L. Linsky, S. Serio (1993), pp. 113-+

B. T. Tsurutani, W. D. Gonzalez, G. S. Lakhina, S. Alex, Journal of Geophysical Research (Space Physics) 108, 1268 (2003), doi:10.1029/2002JA009504

A. Veronig, M. Temmer, A. Hanslmeier, W. Otruba, M. Messerotti, Astron. Astrophys. 382, 1070 (2002a), arXiv:astro-ph/0207234 doi:10.1051/0004-6361:20011694

A. Veronig, B. Vršnak, B. R. Dennis, M. Temmer, A. Hanslmeier, J. Magdalenić, Astron. Astrophys. 392, 699 (2002b), arXiv:astro-ph/0207217 doi:10.1051/0004-6361:20020947

A. M. Veronig, J. C. Brown, B. R. Dennis, R. A. Schwartz, L. Sui, A. K. Tolbert, Astrophys. J. 621, 482 (2005), doi:10.1086/427274

N. Vilmer, A. L. MacKinnon, G. J. Hurford, Space Science Reviews (2010), arXiv: XXX

B. Vršnak, D. Sudar, D. Ruždjak, Astron. Astrophys. 435, 1149 (2005), doi:10.1051/0004-6361:20042166

Y. Wang, J. Zhang, Astrophys. J. 665, 1428 (2007), 0808.2976 doi:10.1086/519765

M. S. Wheatland, Astrophys. J. Lett. 536, L109 (2000), arXiv : astro-ph/0005121 doi:10.1086/312739

M. S. Wheatland, Solar Phys. 203, 87 (2001)

M. S. Wheatland, Astrophys. J. 609, 1134 (2004), arXiv:astro-ph/0403613 doi:10.1086/421261

M. S. Wheatland, Astrophys. J. 679, 1621 (2008), 0802.3931 doi:10.1086/587871

M. S. Wheatland, Astrophys. J. 710, 1324 (2010), 1001.1464 doi:10.1088/0004-637X/710/2/1324

M. S. Wheatland, S. Glukhov, Astrophys. J. 494, 858 (1998), doi:10.1086/305245

M. S. Wheatland, Y. E. Litvinenko, Solar Phys. 211, 255 (2002)

M. S. Wheatland, P. A. Sturrock, J. M. McTiernan, Astrophys. J. 509, 448 (1998), doi:10.1086/306492

S. M. White, K. Arzner, S. Christe, F. Farnik, K.-L. Klein, M. R. Kundu, Z. Ning, J.-P. Raulin, A. V. R. Silva Valio, P. Saint-Hilaire, G. Trottet, A. Warmuth, Space Science Reviews (2010), arXiv: XXX

J. P. Wild, S. F. Smerd, A. A. Weiss, ARAA 1, 291 (1963), doi:10.1146/annurev.aa.01.090163.001451

J. A. Willick, Astrophys. Suppl. 92, 1 (1994), doi:10.1086/191957

T. N. Woods, F. G. Eparvier, J. Fontenla, J. Harder, G. Kopp, W. E. McClintock, G. Rottman, B. Smiley, and M. Snow, Geophys. Res. Lett. 3110802 (2004).

S. Yashiro, S. Akiyama, N. Gopalswamy, R. A. Howard, Astrophys. J. Lett. 650, L143 (2006), arXiv:astro-ph/0609197 doi:10.1086/508876

S. Yashiro, N. Gopalswamy, G. Michalek, O. C. St. Cyr, S. P. Plunkett, N. B. Rich, R. A. Howard, Journal of Geophysical Research (Space Physics) 109(A18), 7105 (2004), doi:10.1029/2003JA010282

T. Yoshida, S. Tsuneta, Astrophys. J. 459, 342 (1996), doi:10.1086/176897 


\section{Index}

absorption

Earth's atmosphere, 8

abundances, 24

active regions

as source of background radiation, 9

association with major flares, 4

association with microflares, 7

illustration, 7

non-flaring X-ray emission, 15, 16

simple structures, 4

active-region transient brightenings

identification with microflares, 9

illustration, 24

albedo, 22

correction, 23

illustration, 22

dip in spectrum, 24

microflare spectra, 23

ARTB, 4

big-flare syndrome, 5

microflares, 27

bremsstrahlung, 23

thick-target, 2

caveats

emission-measure weighting, 17

energy scale for occurrence distribution, 14

gaps in time series, 20

nature of low-energy cutoff, 24

non-scalable properties, 5

RHESSI rotation axis, 9

cellular automaton, 21

Chianti, 24

chromospheric evaporation, 10

coronal heating

nanoflares, 3

coronal mass ejections (CMEs), 6

and microflares, 6

and soft-hard-harder pattern, 7

Coulomb collisions, 2

distributions

log-normal, 17

power law, 31

skew-Laplace, 32

Weibull, 17, 32

earthquakes, 18

electrons

accelerated

in microflares, 12

beams, 28,29

nonthermal, 22

early impulsive events, 23

spectrum

low-energy cutoff, 24

selection effects, 22

total energy, 29

emission measure

and Neupert effect, 28

correlation with temperature

broad parameter ranges, 26

GOES, 26

illustration, 25

RHESSI, 26

experimental bias

DEM weighting, 25

Fe lines

in microflare spectra, 9

filling factor

distribution functions, 29

flare (individual)

SOL1859-09-01T11:18 (pre-GOES), 4

SOL2002-05-06T09:01 (A8.0)

illustration, 10

SOL2003-10-28T11:10 (X17.2)

TSI signature, 30

SOL2003-11-04T19:53 (X17.4)

most energetic event, 4

SOL2006-11-06T08:32 (B1)

illustration, 9

SOL2006-11-06T22:40 (A1)

illustration, 9

SOL2006-11-06T22:43 (A3)

illustration, 9

flare classification, 4

$\mathrm{H} \alpha, 7$

table, 4

flare frequency distributions, 2, 16, 28

active-region dependence, 18

and natural limits, 28

and Poisson distribution, 21

background corrections, 17

biases, 18

break energy, 24

durations, 19

illustration, 4

microflare comparison, 30

multivariate analysis, 19

nanoflare range, 30

nanoflares

inconsistency, 30

nonthermal, 30

observational range, 3

observations of power laws

table, 16

observed parameters

compared with derived, 17

normalized to specific photon energy, 
sensitivity limit, 17

physical range, 3

stellar flares, 18

temporal confusion, 17

total power, 3

why a power law?, 21

X-rays, 17

flare models

avalanche, 20, 32

cellular automaton, 21

self-organized criticality (SOC), 2, 20

thick-target, 29

flare types

early impulsive, 23

white light

energy, 29

flares

association with CMEs

illustration, 6

Carrington, 4

classification, 4

table, 4

$\mathrm{H} \alpha, 7$

scale invariance, 18

stellar

occurrence distributions, 18

"super flares", 18

X-ray fluences, 18

footpoints

microflares, 10

HXR, 10

illustration, 10, 12

microwave emission, 12

frequency

occurrence, 2

gamma-rays

GOES

$\pi^{0}$-decay in microflares, 5

classification, 4

illustration, 3

gradual phase

in microflares, 27

Gutenberg-Richter law, 18

gyrosynchrotron emission

microflares, 12

rarity in network flares, 15

hard X-rays

and with type III bursts, 12

flux saturation, 17

microflares, 9

quiet Sun, 14, 15

and nanoflares, 16

illustration, 14

RHESSI

microflare observations, 3

spectra

soft-hard-soft, 22

HXIS, 8 imaging

microflares, 8

inverse problem

and microflare hard X-ray spectra, 23

microflare imaging, 10

ionization state, 24

jets

and loop brightenings, 13

recurring, 13

X-ray, 13

loops

$\mathrm{H} \alpha$ footpoints in microflares, 10

in microflares, 10

microflare

lengths, 10

loss of equilibrium, 21

low-energy cutoff, 23

in microflare hard X-ray spectra, 23, 29

magnetic field

XBP distribution, 14

magnetic structures

jets and open fields, 13

microflare image morphology, 10

Malmquist bias, 30

master equation, 21

microflares, 7

active-region association, 8

albedo correction, 23

and CMEs, 6

and $\mathrm{MeV}$ electrons, 12

and open field lines, 13

and type III bursts, 12

association with magnetic neutral line, 9

energy distributions

illustration, 24

energy estimates, 29

event energies

fluence determination, 18

Fe line, 9

gyrosynchrotron emission, 12

hard X-ray

discovery, 4

illustration, 13, 14

imaging, 8

jet association, 13

microwave emission, 12

non-thermal HXR, 9

observational bias towards low activity, 9

radio emission, 12

solar-cycle dependence, 9

steep hard X-ray spectra, 10

thermal spectrum, 24

thermal/nonthermal relationship, 26

$\mathrm{X}$-ray spectral parameters, 22

nanoflares, 3, 16

and cellular automata, 21 
and coronal heating, 16

energy range, 4

EUV, 30

Parker's hypothesis, 3

problem of energy scale, 14

selection effects, 30

network flares, 15

Neupert effect, 22, 28

in microflares, 27

Nobeyama radioheliograph, 12

observatories

NoRH, 12

occulted sources, 23, 24

illustration, 22, 23

Parker's nanoflare mechanism, 3, 4

Parker, E. N., 30

probability distribution function, 2

quiet Sun, 14

absence of microflares, 8

radio emission

gyrosynchrotron

microflares, 12

type III burst, 6, 12

and jets, 13

EUV jets, 13

illustration, 14

waiting time distribution, 20

reconnection, 13

relaxation oscillator, 21

RHESSI, 2

attenuating shutters, 8

and deadtime, 28

and microflares, 8

HXR from quiet Sun, 15

offpointing, 15

rotation axis and imaging, 9

satellites

CGRO, 2, 8

BATSE, 4

GOES, 3, 4, 7, 27

GRANAT

WATCH, 2

Hinode, 13

ICE, 2,19

ISEE-3, 2, 19

OSO $-3,2$

OSO-7, 2

RHESSI, 2

Skylab, 14

SMM, 2

HXIS, 8

HXRBS, 4, 27

SOHO , 2, 14

SORCE

TIM, 30
TRACE , 2, 13, 14

WIND

3DP, 7

WAVES, 14

Yohkoh, 2, 8, 28

BCS, 25

HXT, 8

SXT, 4, 24, 25, 28

scale invariance, 19, 21

self-organized critical state, 2, 19-21

shocks

interplanetary, 6

Skylab, 14

soft X-rays

Neupert effect

microflares, 22

soft-hard-soft, 22

solar energetic particles (SEPs), 6

and microflares, 6

gradual events, 6

impulsive events, 6

sunspots

and network field, 15

syndromes

big-flare, 5

microflares, 27

trapping

and microwaves, 12

TSI, 30

visibilities, 28

waiting-time distribution, 19, 20

time-varying Poisson occurrence, 20

type III bursts, 20

white-light flares

and HXR, 29

big-flare syndrome, 5

emission patches, 4

X-ray bright points (XBP), 14

and basal coronal emission, 15

and jets, 15

and surface convective flows, 15

footpoints, 14

hard X-rays, 15

loops, 14

spatial distribution, 14

Yohkoh

Soft X-ray Telescope (SXT), 8, 24 